\title{
HARDY INEQUALITIES WITH BEST CONSTANTS ON FINSLER METRIC MEASURE MANIFOLDS
}

\author{
WEI ZHAO
}

\begin{abstract}
The paper is devoted to weighted $L^{p}$-Hardy inequalities with best constants on Finsler metric measure manifolds. There are two major ingredients. The first, which is the main part of this paper, is the Hardy inequalities concerned with distance functions in the Finsler setting. In this case, we find that besides the flag curvature, the Ricci curvature together with two non-Riemannian quantities, i.e., reversibility and S-curvature, also play an important role. And we establish the optimal Hardy inequalities not only on noncompact manifolds, but also on closed manifolds. The second ingredient is the Hardy inequalities for Finsler $p$-sub/superharmonic functions, in which we also investigate the existence of extremals and the Brezis-Vázquez improvement.
\end{abstract}

\section{INTRODUCTION}

The classical Hardy inequality states that for any $p>1$,

$$
\int_{\mathbb{R}^{n}}|\nabla u|^{p} d x \geq\left(\frac{|n-p|}{p}\right)^{p} \int_{\mathbb{R}^{n}} \frac{|u|^{p}}{|x|^{p}} d x, \forall u \in C_{0}^{\infty}\left(\mathbb{R}^{n} \backslash\{\mathbf{0}\}\right)
$$

where $\left(\frac{|n-p|}{p}\right)^{p}$ is sharp (see for instance Hardy et al. [16]). It is well-known that Hardy inequalities play a prominent role in the theory of linear and nonlinear partial differential equations. For example, they are useful to investigate the stability of solutions of semilinear elliptic and parabolic equations, the existence and asymptotic behavior of the heat equations and the stability of eigenvalues in elliptic problems. See e.g. $[4,7,10,14,31,36,37]$ and references therein.

In recent years, a great deal of effort has been devoted to the study of Hardy inequalities in curved spaces. As far as we know, Carron [8] was the first who studied weighted $L^{2}$-Hardy inequalities on complete, non-compact Riemannian manifolds. On one hand, inspired by [8], a systematic study of the Hardy inequality is carried out by Berchio, Ganguly and Grillo [6], D'Ambrosio and Dipierro [13], Kombe and Özaydin [23,24], Yang, Su and Kong [41] in the Riemannian setting (where the canonical Riemannian measure is used). On the other hand, Kristály and Repovš [19], Kristály and Szakál [21] and Yuan, Zhao and Shen [43] studied quantitative Hardy inequalities on Finsler manifolds with vanishing S-curvature while Mercaldo, Sano and Takahshi [25] investigated $L^{p}$-Hardy inequalities in reversible Minkowski spaces (where the Busemann-Hausdorff measure is used).

In this paper, a Finsler metric measure manifold is a Finsler manifold equipped with a smooth measure. Thus, all the aforementioned spaces are special cases of Finsler metric measure manifolds. However, up to now, limited work has been done in the study of Hardy inequalities on general Finsler metric measure manifolds. A key issue is that two non-Riemannian quantities have a great effect on Hardy inequalities in such a setting, as

- reversibility;

- $S$-curvature induced by the given measure.

In order to emphasize the influence, we present a simple example.

2010 Mathematics Subject Classification. Primary 26D10, Secondary 53C60, 53C23.

Key words and phrases. Hardy inequality, best constant, Finsler manifold, Riemannian manifold, metric measure manifold, $p$-Laplacian, subharmonic function. 
Example 1 ( $[18,20])$. Consider the Funk metric measure manifold $\left(M, F, d \mathfrak{m}_{B H}\right)$, where $M:=\mathbb{B}_{\mathbf{0}}(1)$ is the unit ball centered at $\mathbf{0}$ in $\mathbb{R}^{n}$,

$$
F(x, y):=\frac{\sqrt{|y|^{2}-\left(|x|^{2}|y|^{2}-\langle x, y\rangle^{2}\right)}}{1-|x|^{2}}+\frac{\langle x, y\rangle}{1-|x|^{2}},
$$

and $d \mathfrak{m}_{B H}$ is the Busemann-Hausdorff measure.

In this case, the Euclidean quantities $|\nabla u(x)|,|x|$ and $d x$ from (1.1) are naturally replaced by the coFinslerian norm of the differential $F^{*}(d u)$, the Finsler distance function $d_{F}(\mathbf{0}, x)$, and the measure $d \mathfrak{m}_{B H}$, respectively. In spite of the fact that $(M, F)$ is simply connected, forward complete and has constant flag curvature $-\frac{1}{4}$, the Hardy inequality fails:

$$
\inf _{u \in C_{0}^{\infty}(M) \backslash\{0\}} \frac{\int_{M} F^{* 2}(d u) d \mathfrak{m}_{B H}}{\int_{M} \frac{u^{2}}{d_{F}^{2}(\mathbf{0}, x)} d \mathfrak{m}_{B H}}=0 .
$$

We remark that $\left(M, F, d \mathfrak{m}_{B H}\right)$ has infinite reversibility and non-vanishing $S$-curvature.

The purpose of this paper is to investigate weighted $L^{p}$-Hardy inequalities with best constants on general Finsler metric measure manifolds. In order to state our main results, we introduce and recall some notations (for details, see Section 2). A triple $(M, F, d \mathfrak{m})$ always denotes a Finsler metric measure manifold, i.e., $(M, F)$ is a Finsler manifold endowed with a smooth measure $d \mathfrak{m}$. Given a Finsler metric measure manifold $(M, F, d \mathfrak{m})$, the reversibility, introduced by Rademacher [32], is defined as

$$
\lambda_{F}:=\sup _{x \in M} \lambda_{F}(x), \text { where } \lambda_{F}(x)=\sup _{y \in T_{x} M \backslash\{0\}} \frac{F(x,-y)}{F(x, y)} .
$$

Obviously, $\lambda_{F} \geq 1$ with equality if and only if $F$ is reversible (i.e., symmetric). Riemannian metrics are always reversible, but there are infinitely many non-reversible Finsler metrics (e.g. see Example 1). Furthermore, the distance function $d_{F}$ induced by $F$ is usually asymmetric (i.e., $d_{F}\left(x_{1}, x_{2}\right) \neq d_{F}\left(x_{2}, x_{1}\right)$ ) unless $\lambda_{F}=1$. Given a point $o \in M$, we use the following notations throughout this paper:

$$
r_{+}(x):=d_{F}(o, x), r_{-}(x):=d_{F}(x, o) .
$$

Since there is no canonical measure on a Finsler manifold, various measures can be introduced whose behavior may be genuinely different. A measure $d \mathfrak{m}$ induces two further geometric quantities $\tau$ and $\mathbf{S}$, see Shen [35], which are the so-called distortion and S-curvature, respectively. More precisely, if $d \mathfrak{m}:=\sigma(x) d x^{1} \wedge \ldots \wedge d x^{n}$ in some local coordinate $\left(x^{i}\right)$, for any $y \in T_{x} M \backslash\{0\}$, let

$$
\tau(y):=\log \frac{\sqrt{\operatorname{det} g_{i j}(x, y)}}{\sigma(x)}, \quad \mathbf{S}(y):=\left.\frac{d}{d t}\right|_{t=0}\left[\tau\left(\dot{\gamma}_{y}(t)\right)\right],
$$

where $g_{y}=\left(g_{i j}(x, y)\right)$ is the fundamental tensor induced by $F$ and $t \mapsto \gamma_{y}(t)$ is the geodesic starting at $x \in M$ with $\dot{\gamma}_{y}(0)=y \in T_{x} M$. Although both the distortion and the S-curvature vanish on every Riemannian manifold endowed with the canonical Riemannian measure, these two quantities have already appeared in Riemannian metric measure manifolds, just in different forms.

Example 2. Let $\left(M, g, e^{-f} d \operatorname{vol}_{g}\right)$ denote a Riemannian metric measure manifold, i.e., $(M, g)$ is a Riemannian manifold, $f \in C^{\infty}(M)$ and $d \operatorname{vol}_{g}$ is the canonical Riemannian measure. Note that $\left(M, g, e^{-f} d \operatorname{vol}_{g}\right)$ can be viewed as a Finsler metric measure space $\left(M, \sqrt{g}, e^{-f} d \operatorname{vol}_{g}\right)$. Therefore, for any $x \in M$, one has

$$
\tau(y)=f(x), \mathbf{S}(y)=g(y, \nabla f), \forall y \in T_{x} M .
$$

Thus, the S-curvature does not vanish unless $f$ is a constant.

Let $r(\cdot)$ be the distance function from a fixed point $o \in M$ induced by $g$. Then the assumption that $\partial_{r} f \geq 0$ along all the minimal geodesics from o is equivalent to that the $S$-curvature is nonnegative along all minimal geodesics from o. Clearly, the Euclidean space equipped with the Gaussian measure $\left(\mathbb{R}^{n},(\cdot, \cdot), \frac{1}{\sqrt{2 \pi}} e^{-\frac{1}{2}\|x\|^{2}} d x\right)$ satisfies this assumption if $o$ is the origin. 
The S-curvature must vanish if it is non-positive (or nonnegative) on a reversible Finsler metric measure manifold. Hence, $\mathbf{S} \geq 0$ (or $\mathbf{S} \leq 0$ ) is a strong condition. Inspired by Example 2, we introduce a weaker assumption: given a point $o \in M$, we say $\mathbf{S}_{o}^{+} \geq 0$ (resp., $\mathbf{S}_{o}^{-} \geq 0$ ) if the S-curvature is nonnegative along all minimal geodesics from (resp., to) o. And $\mathbf{S}_{o}^{ \pm} \leq 0$ are defined similarly. We remark that $\mathbf{S}_{o}^{+} \leq 0$ is not equivalent to $\mathbf{S}_{o}^{-} \geq 0$ in the irreversible case. For instance, the Funk metric measure manifold in Example 1 satisfies $\mathbf{S}_{o}^{+}=\mathbf{S}_{o}^{-}=\frac{n+1}{2}$ for every point $o \in M$.

In Finsler geometry the flag curvature is a geometric quantity analogous to the sectional curvature. Let $P:=\operatorname{Span}\{y, v\} \subset T_{x} M$ be a plane. The flag curvature is defined by

$$
\mathbf{K}(y, v):=\frac{g_{y}\left(R_{y}(v), v\right)}{g_{y}(y, y) g_{y}(v, v)-g_{y}^{2}(y, v)},
$$

where $R_{y}$ is the Riemannian curvature of $F$. A Finsler metric measure manifold $(M, F, d \mathfrak{m})$ is called a Cartan-Hadamard measure manifold if $(M, F)$ is a simply connected forward complete Finsler manifold with $\mathbf{K} \leq 0$.

Our first result reads as follows.

Theorem 1.1. Let $(M, F, d \mathfrak{m})$ be an n-dimensional Cartan-Hadamard measure manifold. Given $o \in M$, set $r_{-}(x):=d_{F}(x, o)$. If $\mathbf{S}_{o}^{-} \geq 0$, then for any $p, \beta \in \mathbb{R}$ with $p \in(1, n)$ and $\beta>-n$, we have

$$
\int_{M} r_{-}^{\beta+p} \max \left\{F^{* p}( \pm d u)\right\} d \mathfrak{m} \geq\left(\frac{n+\beta}{p}\right)^{p} \int_{M} r_{-}^{\beta}|u|^{p} d \mathfrak{m}, \forall u \in C_{0}^{\infty}(M) .
$$

In particular, the constant $\left(\frac{n+\beta}{p}\right)^{p}$ is sharp if $\lambda_{F}=1$.

If $F$ is reversible, then $F^{*}( \pm d u)=F(\nabla u)$, i.e., the norm of the gradient of $u$. Therefore, Theorem 1.1 implies the classical Hardy inequality $(1.1)$ for $p \in(1, n)$, the Hardy inequality on Riemannian manifolds (cf. Yang et al. [41, Theorem 3.1] and D'Ambrosio et al. [13, Theorem 6.5]) and the quantitative Hardy inequality on Finsler manifolds (cf. Kristály et al. [19]). In particular, for the Funk metric measure manifold in Example 1, the inequality above yields (compare (1.2))

$$
\inf _{u \in C_{0}^{\infty}(M) \backslash\{0\}} \frac{\int_{M} \max \left\{F^{* 2}( \pm d u)\right\} d \mathfrak{m}_{B H}}{\int_{M} \frac{u^{2}}{d_{F}^{2}(x, \mathbf{0})} d \mathfrak{m}_{B H}} \geq\left(\frac{n-2}{2}\right)^{2} .
$$

Furthermore, Theorem 1.1 remains valid under the weaker assumptions. See Theorem 3.8 below. The counterpart of Theorem 1.1 is as follows.

Theorem 1.2. Let $(M, F, d \mathfrak{m})$ be an $n$-dimensional Cartan-Hadamard measure manifold. Given o $\in M$, set $r_{+}(x):=d_{F}(o, x)$. If $\mathbf{S}_{o}^{+} \leq 0$, then for any $p, \beta \in \mathbb{R}$ with $p>n>-\beta$, we have

$$
\int_{M} r_{+}^{\beta+p} \max \left\{F^{* p}( \pm d u)\right\} d \mathfrak{m} \geq\left(\frac{n+\beta}{p}\right)^{p} \int_{M} r_{+}^{\beta}|u|^{p} d \mathfrak{m}, \forall u \in C_{0}^{\infty}(M) .
$$

In particular, the constant $\left(\frac{n+\beta}{p}\right)^{p}$ is sharp if $(M, F, d \mathfrak{m})$ satisfies

$$
\lambda_{F}=1, \mathbf{K}=0, \mathbf{S}_{o}^{+}=0 .
$$

We discuss the spaces with (1.3) briefly. Since a flat Riemannian Cartan-Hadamard manifold is always isometric to a Euclidean space, we get nothing new in the Riemannain setting. However, it is another story in the Finsler setting. There are plenty of Finsler metric measure manifolds satisfying (1.3) which are not isometric to each other (see Example 4 below). Hence, Theorem 1.2 provides a number of new models on which the inequality above is optimal. Moreover, this theorem can be extended to a more general case. See Theorem 3.9 below.

We also have a logarithmic Hardy inequality. 
Theorem 1.3. Let $(M, F, d \mathfrak{m})$ be an n-dimensional reversible Cartan-Hadamard measure manifold. Given $o \in M$, set $r(x):=d_{F}(x, o)$ and $B_{o}(R):=\{x \in M: r(x)<R\}$. If $\mathbf{S}_{o}^{+} \leq 0$, for any $R, p, \beta \in \mathbb{R}$ with $R>0, p \in(1, n]$ and $\beta<-1$, we have

$$
\int_{B_{o}(R)}\left[\log \left(\frac{R}{r}\right)\right]^{p+\beta} F^{* p}(d u) d \mathfrak{m} \geq\left(\frac{|\beta+1|}{p}\right)^{p} \int_{B_{o}(R)}\left[\log \left(\frac{R}{r}\right)\right]^{\beta} \frac{|u|^{p}}{r^{p}} d \mathfrak{m},
$$

for any $u \in C_{0}^{\infty}\left(B_{o}(R)\right)$. In particular, the constant $\left(\frac{|\beta+1|}{p}\right)^{p}$ is sharp.

From Theorem 1.3, one can easily derive the logarithmic Hardy inequalities on Euclidean spaces, Riemannian Cartan-Hadamard manifolds and reversible Minksowski spaces, respectively (e.g. see [12,13, 25]). Moreover, Theorem 1.3 can be generalized to the irreversible case. See Theorem 3.10 below.

Now we turn to consider the Hardy inequalities concerned with the Ricci curvature. Although this problem is also genuinely new in the Riemannian framework, we prefer to study it in the context of Finsler geometry.

Given an $n$-dimensional Finsler metric measure manifold $(M, F, d \mathfrak{m})$, by means of the flag curvature, one can define the Ricci curvature Ric in the usual way. The weighted Ricci curvature $\mathbf{R i c}_{N}$, introduced in Ohta and Sturm [27], is defined as follows: given $N \in[n, \infty]$, for any unit vector $y \in T M$,

$$
\boldsymbol{R i c}_{N}(y)= \begin{cases}\boldsymbol{\operatorname { R i c }}(y)+\left.\frac{d}{d t}\right|_{t=0} \mathbf{S}\left(\gamma_{y}(t)\right)-\frac{\mathbf{S}^{2}(y)}{N-n}, & \text { for } N \in(n, \infty), \\ \lim _{L \downarrow n} \operatorname{Ric}_{L}(y), & \text { for } N=n, \\ \boldsymbol{\operatorname { R i c }}(y)+\left.\frac{d}{d t}\right|_{t=0} \mathbf{S}\left(\gamma_{y}(t)\right), & \text { for } N=\infty .\end{cases}
$$

The weighted Ricci curvature has an important influence on the geometry of Finsler manifolds. See Ohta [28-30], etc. for surveys. Now we state the result as follows.

Theorem 1.4. Let $(M, F, d \mathfrak{m})$ be an $n$-dimensional forward complete Finsler metric measure manifold with $\mathbf{R i c}_{N} \geq 0$, where $N \in[n, \infty)$. Given $o \in M$, define $r_{+}(x):=d_{F}(o, x)$ and $r_{-}(x):=d_{F}(x, o)$.

(1) Given $p, \beta \in \mathbb{R}$ with $p>N$ and $\beta<-N$, for any $u \in C_{0}^{\infty}(M \backslash\{o\})$, we have

$$
\int_{M \backslash\{o\}} r_{+}^{\beta+p} \max \left\{F^{* p}( \pm d u)\right\} d \mathfrak{m} \geq\left(\frac{|N+\beta|}{p}\right)^{p} \int_{M \backslash\{o\}} r_{+}^{\beta}|u|^{p} d \mathfrak{m} .
$$

(2) Given $p, \beta \in \mathbb{R}$ with $1<p<N$ and $\beta<-N$, for any $u \in C_{0}^{\infty}(M \backslash\{o\})$, we have

$$
\int_{M \backslash\{o\}} r_{-}^{\beta+p} \max \left\{F^{* p}( \pm d u)\right\} d \mathfrak{m} \geq\left(\frac{|N+\beta|}{p}\right)^{p} \int_{M \backslash\{o\}} r_{-}^{\beta}|u|^{p} d \mathfrak{m} .
$$

In particular, the constants are sharp in (1.4) and (1.5) if $\lambda_{F}=1, N=n$ and $p+\beta>-n$.

Clearly, the inequality (1.4) implies the classical Hardy inequality (1.1) for $p>n$. We remark that the manifold $M$ in Theorem 1.4 is unnecessarily noncompact. In fact, $M$ must be closed if $\mathbf{R i c}_{N}$ is bounded below by a positive number (cf. Ohta [28]). On the other hand, Ric $\mathbf{N}_{N}$ has a close relation with the Bakry-Émery Ricci tensor. More precisely, for a Riemannian metric measure manifold $\left(M, g, e^{-f} d \operatorname{vol}_{g}\right)$, Example 2 furnishes

$$
\boldsymbol{R i c}_{N+n}=\mathbf{R i c}_{N}^{\mathrm{BÉ}}, \forall N \in(0,+\infty],
$$

where $\mathbf{R i c}_{N}^{\mathrm{BE}}$ denotes the $N$-Bakry-Émery Ricci tensor (cf. Wei and Wylie [38]). As a consequence, Theorem 1.4 inspires the following result.

Theorem 1.5. Let $\left(M, g, e^{-f} d \mathrm{vol}_{g}\right)$ be an $n$-dimensional closed Riemannian metric measure manifold with $\mathbf{R i c}_{\infty}^{B E ́} \geq 0$. Given $o \in M$, let $r$ denote the distance function from o. Suppose $\partial_{r} f \geq 0$ along all the minimal geodesics from $o$. Thus, for any $p \in(1, n) \cup(n, \infty)$ and $\beta<-n$ with $p+\beta>-n$, we have

$$
\int_{M} r^{\beta+p}|\nabla u|^{p} e^{-f} d \operatorname{vol}_{g} \geq\left(\frac{|n+\beta|}{p}\right)^{p} \int_{M} r^{\beta}|u|^{p} e^{-f} d \operatorname{vol}_{g}, \forall u \in \mathfrak{C}^{\infty}(M, o),
$$


where $\mathfrak{C}^{\infty}(M, o):=\left\{u \in C^{\infty}(M): u(o)=0\right\}$. In particular, $\left(\frac{|n+\beta|}{p}\right)^{p}$ is sharp with respect to $\mathfrak{C}^{\infty}(M, o)$.

On a closed manifold $M$, the Hardy inequality (1.1) fails for $u \in C_{0}^{\infty}(M)$ because in this case the constant functions belong to $C_{0}^{\infty}(M)$. Theorem 1.5 then indicates what kind of function (1.1) remains valid for. See Theorem 3.16 below for a Finsler version of the theorem above.

We note that Theorems 1.1-1.4 can be established on Riemannian metric measure manifolds and backward complete Finsler metric measure manifolds; we leave the formulation of such statements to the interested reader.

The paper is organized as follows. Section 2 is devoted to preliminaries on Finsler geometry. The proofs of Theorem 1.1-1.5 are given in Section 3, while the Hardy inequalities for Finsler $p$-sub/superharmonic functions are discussed in Section 4. We devote Appendix A and B to some necessary tools which are useful to prove Theorems 1.3 and 1.5.

\section{Preliminaries}

2.1. Elements from Finsler geometry. In this section, we recall some definitions and properties from Finsler geometry; for details see Bao, Chern and Shen [5], Ohta and Sturm [27] and Shen [34,35].

2.1.1. Finsler manifolds. Let $M$ be a $n$-dimensional connected smooth manifold and $T M=\bigcup_{x \in M} T_{x} M$ be its tangent bundle. The pair $(M, F)$ is a Finsler manifold if the continuous function $F: T M \rightarrow[0, \infty)$ satisfies the conditions:

(a) $F \in C^{\infty}(T M \backslash\{0\})$;

(b) $F(x, \lambda y)=\lambda F(x, y)$ for all $\lambda \geq 0$ and $(x, y) \in T M$;

(c) $g_{y}:=g_{i j}(x, y)=\left[\frac{1}{2} F^{2}\right]_{y^{i} y^{j}}(x, y)$ is positive definite for all $(x, y) \in T M \backslash\{0\}$, where $F(x, y):=$ $F\left(\left.y^{i} \frac{\partial}{\partial x^{i}}\right|_{x}\right)$.

The quantity $g_{y}:=\left(g_{i j}(x, y)\right)$ is called the fundamental tensor. It can be defined at $y=0$ if and only if $F$ is Riemannian, in which case $g$ is independent of $y$, i.e., $g=\left(g_{i j}(x)\right)$. The Euler theorem yields $F^{2}(x, y)=g_{y}(y, y)=g_{i j}(x, y) y^{i} y^{j}$. Moreover, we have a Cauchy-Schwartz inequality

$$
g_{y}(y, w) \leq F(x, y) F(x, w), \forall y, w \in T_{x} M,
$$

with equality if and only if $w=k y$ for $k \geq 0$.

Set $S_{x} M:=\left\{y \in T_{x} M: F(x, y)=1\right\}$ and $S M:=\cup_{x \in M} S_{x} M$. The reversibility $\lambda_{F}$ (cf. Rademacher [32]) and the uniformity constant $\Lambda_{F}$ (cf. Egloff [15]) of $(M, F)$ are defined as follows:

$$
\lambda_{F}:=\sup _{y \in S M} F(-y), \Lambda_{F}:=\sup _{X, Y, Z \in S M} \frac{g_{X}(Y, Y)}{g_{Z}(Y, Y)} .
$$

Clearly, $\Lambda_{F} \geq \lambda_{F}^{2} \geq 1$. In particular, $\lambda_{F}=1$ if and only if $F$ is reversible (i.e., symmetric), while $\Lambda_{F}=1$ if and only if $F$ is Riemannian. For convenience, we introduce the reversibility of a subset $U \subset M$, i.e.,

$$
\lambda_{F}(U):=\sup _{y \in S U} F(-y), \text { where } S U:=\cup_{x \in U} S_{x} M .
$$

Thus, $\lambda_{F}(M)=\lambda_{F}$, and $\lambda_{F}(U)$ is finite if $\bar{U}$ is compact.

The dual Finsler metric $F^{*}$ of $F$ on $M$ is defined by

$$
F^{*}(x, \xi):=\sup _{y \in T_{x} M \backslash\{0\}} \frac{\xi(y)}{F(x, y)}, \forall \xi \in T_{x}^{*} M,
$$

which is a Finsler metric on $T^{*} M$. Let $g_{\xi}^{*}$ be the fundamental tensor of $F^{*}$. Then Yuan et al. [43, Theorem 3.5] furnishes

$$
F^{* 2}(\xi+\eta) \geq F^{* 2}(\xi)+2 g_{\xi}^{*}(\xi, \eta)+\frac{1}{\Lambda_{F}} F^{* 2}(\eta), \forall \xi, \eta \in T_{x}^{*} M,
$$

where $g_{\xi}^{*}(\xi, \eta):=0$ if $\xi=0$. 
The Legendre transformation $\mathfrak{L}: T M \rightarrow T^{*} M$ is defined by

$$
\mathfrak{L}(X):= \begin{cases}g_{X}(X, \cdot), & \text { if } X \neq 0, \\ 0, & \text { if } X=0 .\end{cases}
$$

In particular, $\mathfrak{L}: T M \backslash\{0\} \rightarrow T^{*} M \backslash\{0\}$ is a diffeomorphism with $F^{*}(\mathfrak{L}(X))=F(X)$, for any $X \in T M$. Now let $f: M \rightarrow \mathbb{R}$ be a $C^{1}$-function on $M$; the gradient of $f$ is defined as $\nabla f=\mathfrak{L}^{-1}(d f)$. Thus, $d f(X)=$ $g_{\nabla f}(\nabla f, X)$. For a non-Riemannian Finsler metric, $\nabla$ is usually nonlinear, i.e., $\nabla(f+h) \neq \nabla f+\nabla h$.

Let $\zeta:[0,1] \rightarrow M$ be a Lipschitz continuous path. The length of $\zeta$ is defined by

$$
L_{F}(\zeta):=\int_{0}^{1} F(\dot{\zeta}(t)) d t
$$

Define the distance function $d_{F}: M \times M \rightarrow[0,+\infty)$ by $d_{F}\left(x_{1}, x_{2}\right):=\inf L_{F}(\sigma)$, where the infimum is taken over all Lipschitz continuous paths $\zeta:[a, b] \rightarrow M$ with $\zeta(a)=x_{1}$ and $\zeta(b)=x_{2}$. Generally, $d_{F}\left(x_{1}, x_{2}\right) \neq d_{F}\left(x_{2}, x_{1}\right)$ unless $F$ is reversible. The forward and backward metric balls $B_{o}^{+}(R)$ and $B_{o}^{-}(R)$ are defined by

$$
B_{o}^{+}(R):=\left\{x \in M: d_{F}(o, x)<R\right\}, B_{o}^{-}(R):=\left\{x \in M: d_{F}(x, o)<R\right\} .
$$

If $F$ is reversible, forward metric balls coincide with backward ones, which are denoted by $B_{o}(R)$.

Given $o \in M$, set $r_{+}(x):=d_{F}(o, x)$ and $r_{-}(x):=d_{F}(x, o)$. Shen [35, Lemma 3.2.3] yields

$$
F\left(\nabla r_{+}\right)=F^{*}\left(d r_{+}\right)=1, F\left(\nabla\left(-r_{-}\right)\right)=F^{*}\left(-d r_{-}\right)=1,
$$

a.e. on $M$. If $F$ is reversible, both $r_{ \pm}(x)$ are denoted by $r(x)$.

A smooth curve $t \mapsto \gamma(t)$ in $M$ is called a (constant speed) geodesic if it satisfies

$$
\frac{d^{2} \gamma^{i}}{d t^{2}}+2 G^{i}\left(\frac{d \gamma}{d t}\right)=0
$$

where

$$
G^{i}(y):=\frac{1}{4} g^{i l}(y)\left\{2 \frac{\partial g_{j l}}{\partial x^{k}}(y)-\frac{\partial g_{j k}}{\partial x^{l}}(y)\right\} y^{j} y^{k}
$$

is the geodesic coefficient. And we always use $\gamma_{y}(t)$ to denote the geodesic with $\dot{\gamma}_{y}(0)=y$.

The Finsler manifold $(M, F)$ is forward complete if every geodesic $t \mapsto \gamma(t), 0 \leq t<1$, can be extended to a geodesic defined on $0 \leq t<\infty$; similarly, $(M, F)$ is backward complete if every geodesic $t \mapsto \gamma(t)$, $0<t \leq 1$, can be extended to a geodesic defined on $-\infty<t \leq 1$. If $(M, F)$ is both forward complete and backward complete, we say $(M, F)$ is complete for short.

The cut value $i_{y}$ of $y \in S_{x} M$ is defined by

$$
i_{y}:=\sup \left\{t: \text { the geodesic }\left.\gamma_{y}\right|_{[0, t]} \text { is globally minimizing }\right\} \text {. }
$$

The injectivity radius at $x$ is defined as $\mathfrak{i}_{x}:=\inf _{y \in S_{x} M} i_{y}$. According to Bao et al. [5] and Yuan et al. [43, Proposition 3.2], if $(M, F)$ is either forward or backward complete, then $\mathfrak{i}_{x}>0$ for any point $x \in M$. The cut locus of $x$ is defined as

$$
\operatorname{Cut}_{x}:=\left\{\gamma_{y}\left(i_{y}\right): y \in S_{x} M \text { with } i_{y}<\infty\right\} .
$$

In particular, $\mathrm{Cut}_{x}$ is closed and has null measure.

2.1.2. Measures and curvatures. A triple $(M, F, d \mathfrak{m})$ is called a FMMM, (i.e., Finsler metric measure manifold $)$ if $(M, F)$ is a Finsler manifold endowed with a smooth measure $d \mathfrak{m}$. In the sequel, the function $x \mapsto \sigma(x)$ denotes the density function of $d \mathfrak{m}$ in a local coordinate system $\left(x^{i}\right)$, i.e.,

$$
d \mathfrak{m}=: \sigma(x) d x^{1} \wedge \cdots \wedge d x^{n} .
$$

The divergence of a vector filed $X$ is defined as

$$
\operatorname{div}(X) d \mathfrak{m}:=d(X\rfloor d \mathfrak{m}) .
$$


If $M$ is compact and oriented, we have the divergence theorem

$$
\int_{M} \operatorname{div}(X) d \mathfrak{m}=\int_{\partial M} g_{\mathbf{n}}(\mathbf{n}, X) d A
$$

where $d A=\mathbf{n}\rfloor d \mathfrak{m}$, and $\mathbf{n}$ is the unit outward normal vector field on $\partial M$, i.e., $F(\mathbf{n})=1$ and $g_{\mathbf{n}}(\mathbf{n}, Y)=0$ for any $Y \in T(\partial M)$.

Given a $C^{2}$-function $f$, set $\mathcal{U}=\left\{x \in M:\left.d f\right|_{x} \neq 0\right\}$. The Laplacian of $f$ is defined on $\mathcal{U}$ by

$$
\Delta f:=\operatorname{div}(\nabla f)=\frac{1}{\sigma(x)} \frac{\partial}{\partial x^{i}}\left(\sigma(x) g^{* i j}\left(\left.d f\right|_{x}\right) \frac{\partial f}{\partial x^{j}}\right),
$$

where $\sigma(x)$ is defined by $(2.5)$ and $\left(g^{* i j}\right)$ is the fundamental tensor of $F^{*}$. As in Ohta et al. [27], we define the distributional Laplacian of $u \in W_{\text {loc }}^{1,2}(M)$ in the weak sense by

$$
\int_{M} v \Delta u d \mathfrak{m}=-\int_{M}\langle\nabla u, d v\rangle d \mathfrak{m} \text { for all } v \in C_{0}^{\infty}(M),
$$

where $\langle\nabla u, d v\rangle:=d v(\nabla u)$ at $x \in M$ denotes the canonical pairing between $T_{x} M$ and $T_{x}^{*} M$.

By (2.5), the distortion $\tau$ and the $S$-curvature $\mathbf{S}$ of $(M, F, d \mathfrak{m})$ are defined as

$$
\tau(y):=\log \frac{\sqrt{\operatorname{det} g_{i j}(x, y)}}{\sigma(x)}, \mathbf{S}(y):=\left.\frac{d}{d t}\right|_{t=0}\left[\tau\left(\dot{\gamma}_{y}(t)\right)\right], \text { for } y \in T_{x} M \backslash\{0\},
$$

where $\sigma(x)$ is defined by $(2.5)$ and $\gamma_{y}(t)$ is a geodesic with $\dot{\gamma}_{y}(0)=y$.

Given a point $o \in M$, we say $\mathbf{S}_{o}^{+} \geq 0$ (resp., $\mathbf{S}_{o}^{+} \leq 0$ ) if the S-curvature is nonnegative (resp., nonpositive) along every minimal geodesic from $o$. On the other hand, we say $\mathbf{S}_{o}^{-} \geq 0$ (resp., $\mathbf{S}_{o}^{-} \leq 0$ ) if the S-curvature is nonnegative (resp., non-positive) along every minimal geodesic to o. In particular, if $F$ is reversible, then $\mathbf{S}_{o}^{+} \geq 0$ (resp., $\left.\mathbf{S}_{o}^{+} \leq 0\right)$ if and only if $\mathbf{S}_{o}^{-} \leq 0$ (resp., $\mathbf{S}_{o}^{-} \geq 0$ ).

The Riemannian curvature $R_{y}$ of $F$ is a family of linear transformations on tangent spaces. More precisely, set $R_{y}:=R_{k}^{i}(y) \frac{\partial}{\partial x^{i}} \otimes d x^{k}$, where

$$
R_{k}^{i}(y):=2 \frac{\partial G^{i}}{\partial x^{k}}-y^{j} \frac{\partial^{2} G^{i}}{\partial x^{j} \partial y^{k}}+2 G^{j} \frac{\partial^{2} G^{i}}{\partial y^{j} \partial y^{k}}-\frac{\partial G^{i}}{\partial y^{j}} \frac{\partial G^{j}}{\partial y^{k}},
$$

and $G^{i}$ 's are the geodesic constants defined in (2.4).

Let $P:=\operatorname{Span}\{y, v\} \subset T_{x} M$ be a plane. The flag curvature is defined by

$$
\mathbf{K}(y, v):=\frac{g_{y}\left(R_{y}(v), v\right)}{g_{y}(y, y) g_{y}(v, v)-g_{y}^{2}(y, v)} .
$$

The Ricci curvature at $y \neq 0$ is defined by $\operatorname{Ric}(y):=\frac{R_{i}^{i}(y)}{F^{2}(y)}$. According to Ohta et al. [27], given $y \in S M$, the weighted Ricci curvature is defined by

$$
\boldsymbol{R i c}_{N}(y)= \begin{cases}\boldsymbol{R i c}(y)+\left.\frac{d}{d t}\right|_{t=0} \mathbf{S}\left(\gamma_{y}(t)\right)-\frac{\mathbf{S}^{2}(y)}{N-n}, & \text { for } N \in(n, \infty), \\ \lim _{L \downarrow n} \operatorname{Ric}_{L}(y), & \text { for } N=n, \\ \boldsymbol{\operatorname { R i c }}(y)+\left.\frac{d}{d t}\right|_{t=0} \mathbf{S}\left(\gamma_{y}(t)\right), & \text { for } N=\infty .\end{cases}
$$

In particular, bounding $\mathbf{R i c}_{n}$ from below makes sense only if $\mathbf{S}=0$.

If $(M, F)$ is either forward complete or backward complete, then there exists a polar coordinate system around every point in $M$ (cf. Yuan et al. [43, Proposition 3.2] and Zhao et al. [44, Section 3]). Fixing an arbitrary point $o \in M$, let $(t, y)$ denote the polar coordinate system around $o$ and write

$$
d \mathfrak{m}=: \hat{\sigma}_{o}(t, y) d t \wedge d \nu_{o}(y)
$$

where $t:=r_{+}$and $d \nu_{o}(y)$ is the Riemannian volume measure on $S_{o} M$ induced by $F$.

Since $S_{o} M$ is compact, the integral $\int_{S_{o} M} e^{-\tau(y)} d \nu_{o}(y)$ is finite. Particularly, this integral is equal to the volume of the standard $(n-1)$-unit Euclidean sphere if $d \mathfrak{m}$ is the Busemann-Hausdorff measure (cf. Shen [33] or Zhao et al. [44]). 
For any fixed $y \in S_{o} M$, we have

$$
\Delta t=\frac{\partial}{\partial t} \log \left(\hat{\sigma}_{o}(t, y)\right), \text { for } 0<t<i_{y} .
$$

In particular, Zhao et al. [44, Lemma 3.1] yields

$$
\lim _{r \rightarrow 0^{+}} \frac{\hat{\sigma}_{o}(t, y)}{t^{n-1}}=e^{-\tau(y)}
$$

According to Zhao et al. [44, Theorem 4.3, Remark 5.3, Theorem 3.6], if $\operatorname{Ric}(\nabla t) \geq-(n-1) k^{2}$ and $\mathbf{S}(\nabla t) \geq-h^{2}$, then

$$
\hat{\sigma}_{o}(t, y) \leq e^{-\tau(y)+h^{2} t} \mathfrak{s}_{-k^{2}}^{n-1}(t) \text {, for any } y \in S_{o} M, 0<t<i_{y} .
$$

where $\mathfrak{s}_{-k^{2}}(t)$ is the unique solution to the equation $f^{\prime \prime}(t)-k^{2} f(t)=0$ with $f(0)=0, f^{\prime}(0)=1$.

2.1.3. Reverse Finsler metric measure manifolds. Given a FMMM $(M, F, d \mathfrak{m})$, according to Ohta et al. [27], the reverse of $F$ is defined by $\overleftarrow{F}(x, y):=F(x,-y)$, which is also a Finsler metric. Clearly, $(M, F)$ is forward (resp., backward) complete if and only if $(M, \overleftarrow{F})$ is backward (resp., forward) complete.

In this paper, $(M, \overleftarrow{F}, d \mathfrak{m})$ is called the RFMMM (i.e., reverse Finsler metric measure manifold). Let $\overleftarrow{*}$ denote the geometric quantity $*$ defined by $\overleftarrow{F}$. Then we have

$$
\begin{cases}\overleftarrow{r}_{+}(x)=r_{-}(x), \overleftarrow{r}-(x)=r_{+}(x), & \text { for any } x \in M \\ \overleftarrow{\mathbf{K}}(y, v)=\mathbf{K}(-y, v), \overleftarrow{\mathbf{R i c}}(y)=\mathbf{R i c}(-y), \overleftarrow{\mathbf{S}}(y)=-\mathbf{S}(-y), & \text { for any } y, v \in T M \backslash\{0\} \\ \overleftarrow{\nabla} f=-\nabla(-f), \overleftarrow{\Delta} f=-\Delta(-f), & \text { for any } f \in C^{2}(M)\end{cases}
$$

Remark 1. One can use the polar coordinates to describe of $\mathbf{S}_{o}^{ \pm}$. More precisely, let $(t, y)$ be the polar coordinate system around $o$ in $(M, F, d \mathfrak{m})$. Thus, one has

$$
\left.\left.\mathbf{S}_{o}^{+} \geq 0 \text { (resp., } \leq 0\right) \Longleftrightarrow \mathbf{S}(\nabla t) \geq 0 \text { (resp., } \leq 0\right) \text { for any } y \in S_{o} M, 0<t<i_{y} .
$$

On the other hand, let $(\mathfrak{t}, \mathfrak{y})$ denote the polar coordinate system around $o$ in $(M, \overleftarrow{F}, d \mathfrak{m})$. Then

$$
\left.\mathbf{S}_{o}^{-} \geq 0(\text { resp. }, \leq 0) \Longleftrightarrow \overleftarrow{\mathbf{S}}(\overleftarrow{\nabla} \mathfrak{t}) \leq 0 \text { (resp. }, \geq 0\right) \text { for any } \mathfrak{y} \in \overleftarrow{S_{o} M}, 0<\mathfrak{t}<\overleftarrow{i_{\mathfrak{y}}}
$$

\section{HARdY INEQUALITIES FOR DISTANCE FUNCTIONS}

In this section, we study the Hardy inequalities concerned with distance functions and show Theorem 1.2-Theorem 1.5. Our approach is mainly based on a generalization of the divergence theorem in D'Ambrosio [12] together with the sharp volume comparison for arbitrary measures in Zhao et al. [44]. For simplicity of presentation, we introduce some notations, which are used throughout this paper.

Notations: (1) Let $\Omega$ be a domain (i.e., a connected open subset) in a forward complete Finsler manifold $(M, F)$. We say that $\Omega$ is a natural domain if one of the following statements holds:

(i) $\Omega \subset M$ is a proper domain with smooth non-empty boundary;

(ii) $\Omega=M$ if $M$ is noncompact.

(2) Let $\Omega$ be a natural domain in a forward complete $\operatorname{FMMM}(M, F, d \mathfrak{m})$. We say that a vector filed $X$ belongs to $L_{\text {loc }}^{1}(T \Omega)$ if $\int_{K} F(X) d \mathfrak{m}$ is finite for any compact set $K \subset \Omega$. Given a vector filed $X \in L_{\text {loc }}^{1}(T \Omega)$ and a nonnegative function $f_{X} \in L_{\text {loc }}^{1}(\Omega)$, we say that $f_{X} \leq \operatorname{div} X$ in the weak sense if

$$
\int_{\Omega} u f_{X} d \mathfrak{m} \leq-\int_{\Omega}\langle X, d u\rangle d \mathfrak{m}, \forall u \in C_{0}^{1}(\Omega) \text { with } u \geq 0
$$

where $\langle X, d u\rangle:=d u(X)$.

(3) A quadruple $(M, o, F, d \mathfrak{m})$ is called a PFMMM (i.e., pointed Finsler metric measure manifold) if $(M, F, d \mathfrak{m})$ is a Finsler metric measure manifold and $o$ is a point in $M$. In such a space, $r_{+}\left(\right.$resp., $\left.r_{-}\right)$ 
is always defined as the distance from $o$ (resp., to $o$ ), i.e., $r_{+}(x)=d_{F}(o, x)$ (resp., $\left.r_{-}(x)=d_{F}(x, o)\right)$. In particular, $r_{ \pm}$are denoted by $r$ if $F$ is reversible.

3.1. Main tools. In this subsection, we present the main tools. Inspired by D'Ambrosio [12], we first establish a divergence theorem.

Theorem 3.1. Let $\Omega$ be a natural domain in a forward complete $F M M M(M, F, d \mathfrak{m})$. Let $X \in L_{\text {loc }}^{1}(T \Omega)$ be a vector filed and let $f_{X} \in L_{\mathrm{loc}}^{1}(\Omega)$ be a nonnegative function. Given $p>1$, suppose the following conditions hold:

(i) $f_{X} \leq \operatorname{div} X$ in the weak sense; $\quad$ (ii) $F^{p}(X) / f_{X}^{p-1} \in L_{\text {loc }}^{1}(\Omega)$.

Then we have

$$
\begin{aligned}
p^{p} \int_{\Omega} \frac{F^{p}(-X)}{f_{X}^{p-1}} \max \left\{F^{* p}( \pm d u)\right\} d \mathfrak{m} \geq \int_{\Omega}|u|^{p} f_{X} d \mathfrak{m}, \forall u \in C_{0}^{\infty}(\Omega), \\
p^{p} \int_{\Omega} \frac{F^{p}(X)}{f_{X}^{p-1}} \max \left\{F^{* p}( \pm d u)\right\} d \mathfrak{m} \geq \int_{\Omega}|u|^{p} f_{X} d \mathfrak{m}, \forall u \in C_{0}^{\infty}(\Omega) .
\end{aligned}
$$

Proof. Since the reversibility $\lambda_{F}(K)$ is finite for any compact set $K$, Condition (ii) implies $F^{p}(-X) / f_{X}^{p-1} \in$ $L_{\text {loc }}^{1}(\Omega)$. Now we show (1). It is easy to check $F^{*}(u d u) \leq|u| \max \left\{F^{*}( \pm d u)\right\}$, which together with the assumption, (2.2) and the Hölder inequality yields

$$
\begin{aligned}
& \int_{\Omega}|u|^{p} f_{X} d \mathfrak{m} \leq-\int_{\Omega}\left\langle X, d\left(u^{2}\right)^{p / 2}\right\rangle d \mathfrak{m}=\int_{\Omega} p|u|^{p-2}\langle-X, u d u\rangle d \mathfrak{m} \\
\leq & p \int_{\Omega}|u|^{p-2} F(-X) F^{*}(u d u) d \mathfrak{m} \leq p \int_{\Omega}|u|^{p-1} F(-X) \max \left\{F^{*}( \pm d u)\right\} d \mathfrak{m} \\
\leq & p\left(\int_{\Omega}|u|^{p} f_{X} d \mathfrak{m}\right)^{\frac{p-1}{p}}\left(\int_{\Omega} \frac{F(-X)^{p}}{f_{X}^{p-1}} \max \left\{F^{* p}( \pm d u)\right\} d \mathfrak{m}\right)^{\frac{1}{p}} .
\end{aligned}
$$

Hence, (1) follows. In order to prove (2), note $\langle-X, u d u\rangle \leq F(X) F^{*}(-u d u) \leq|u| F(X) \max \left\{F^{*}( \pm d u)\right\}$. Then the rest of the proof is the same as above.

The following result also plays an important role in establishing the Hardy inequalities.

Lemma 3.2. Let $(M, o, F, d \mathfrak{m})$ be an n-dimensional forward or backward complete PFMMM. Let $\mathfrak{r}$ denote either $r_{+}$or $r_{-}$and set $\mathfrak{B}_{o}(s):=\{x \in M: \mathfrak{r}(x)<s\}$. Then

(1) For any $k \in(-\infty, n)$, we have

$$
\lim _{\epsilon \rightarrow 0^{+}} \int_{\mathfrak{B}_{o}(\epsilon) \backslash\{o\}} \mathfrak{r}^{-k} d \mathfrak{m}=0 .
$$

(2) For any $R>0$, we have

$$
\lim _{\epsilon \rightarrow 0^{+}} \int_{\mathfrak{B}_{o}(R) \backslash \mathfrak{B}_{o}(\epsilon)} \mathfrak{r}^{-n} d \mathfrak{m}=+\infty .
$$

Proof. (i) Suppose $\mathfrak{r}=r_{+}$. Let $(t, y)$ be the polar coordinate system around $o$. In view of (2.8), there exists an $\epsilon_{0} \in\left(0, \mathfrak{i}_{o}\right)$ such that for any $t \in\left(0, \epsilon_{0}\right)$,

$$
\frac{1}{2} e^{-\tau(y)} t^{n-1} \leq \hat{\sigma}_{o}(t, y) \leq 2 e^{-\tau(y)} t^{n-1}, \forall y \in S_{o} M .
$$

Therefore, if $k \in(-\infty, n),(2.7)$ together with the inequality above furnishes

$$
\int_{B_{o}^{+}(\epsilon) \backslash\{o\}} r_{+}^{-k} d \mathfrak{m}=\int_{S_{o} M} d \nu_{o}(y) \int_{0}^{\epsilon} t^{-k} \hat{\sigma}_{o}(t, y) d t \leq \frac{2 \epsilon^{n-k}}{n-k} \int_{S_{o} M} e^{-\tau(y)} d \nu_{o}(y) \rightarrow 0, \text { as } \epsilon \rightarrow 0^{+} .
$$

Similarly, we obtain

$$
\int_{B_{o}^{+}(R) \backslash B_{o}^{+}(\epsilon)} r_{+}^{-n} d \mathfrak{m} \geq \frac{1}{2} \ln \left(\frac{\min \left\{R, \mathfrak{i}_{o}\right\}}{\epsilon}\right) \int_{S_{o} M} e^{-\tau(y)} d \nu_{o}(y) \rightarrow+\infty, \text { as } \epsilon \rightarrow 0^{+} .
$$


(ii) Suppose $\mathfrak{r}=r_{-}$. In this case, we consider the RFMMM $(M, \overleftarrow{F}, d \mathfrak{m})$. Thus, the above results hold for $\overleftarrow{r_{+}}(x):=d_{\overleftarrow{F}}(o, x)$. The assertions then follow from $r_{-}(x)=\overleftarrow{r_{+}}(x)$

Definition 3.3. Let $\Omega \subset M$ be a natural domain in a $\operatorname{FMMM}(M, F, d \mathfrak{m})$. Given $p>1$, for an arbitrary function $f \in C^{\infty}(\Omega)$, the $p$-Laplacian of $f$ is defined as

$$
\Delta_{p} f:=\operatorname{div}\left(F^{p-2}(\nabla f) \nabla f\right) \text { on } \mathcal{U}:=\left\{x \in M:\left.d f\right|_{x} \neq 0\right\} .
$$

Given $c \in \mathbb{R}$, we say that a function $\rho(x) \in W_{\text {loc }}^{1, p}(\Omega)$ satisfies $-c \Delta_{p} \rho \geq 0$ in the weak sense if

$$
c \int_{\Omega} F^{p-2}(\nabla \rho)\langle\nabla \rho, d u\rangle d \mathfrak{m} \geq 0, \forall u \in C_{0}^{1}(\Omega) \text { with } u \geq 0 .
$$

Lemma 3.4. Let $(M, o, F, d \mathfrak{m})$ be a forward complete PFMMM and let $\Omega \subset M$ be a natural domain.

(i) Suppose that $\alpha>0$ and $\beta \in \mathbb{R}$ satisfy the following conditions:

(1) $r_{+}^{(\alpha-1)(p-1)}, r_{+}^{\beta}, r_{+}^{\beta+p} \in L_{\mathrm{loc}}^{1}(\Omega)$;

(2) $-c \Delta_{p}\left(r_{+}^{\alpha}\right) \geq 0$ in the weak sense, where $c=\alpha[(\alpha-1)(p-1)-\beta-1]$.

Then we have

$$
\int_{\Omega} r_{+}^{\beta+p} \max \left\{F^{* p}( \pm d u)\right\} d \mathfrak{m} \geq\left(\vartheta_{\alpha, \beta, p}\right)^{p} \int_{\Omega} r_{+}^{\beta}|u|^{p} d \mathfrak{m}, \forall u \in C_{0}^{\infty}(\Omega)
$$

where

$$
\vartheta_{\alpha, \beta, p}:=\frac{|(\alpha-1)(p-1)-\beta-1|}{p} .
$$

(ii) Suppose that $\alpha>0$ and $\beta \in \mathbb{R}$ satisfy the following conditions:

(1') $r_{-}^{(-\alpha-1)(p-1)}, r_{-}^{\beta}, r_{-}^{\beta+p} \in L_{\mathrm{loc}}^{1}(\Omega)$;

$\left(\mathscr{2}^{\prime}\right)-c \Delta_{p}\left(r_{-}^{-\alpha}\right) \geq 0$ in the weak sense, where $c=\alpha[(\alpha+1)(p-1)+\beta+1]$.

Then we have

$$
\int_{\Omega} r_{-}^{\beta+p} \max \left\{F^{* p}( \pm d u)\right\} d \mathfrak{m} \geq\left(\vartheta_{-\alpha, \beta, p}\right)^{p} \int_{\Omega} r_{-}^{\beta}|u|^{p} d \mathfrak{m}, \forall u \in C_{0}^{\infty}(\Omega),
$$

where

$$
\vartheta_{-\alpha, \beta, p}:=\frac{|(\alpha+1)(p-1)+\beta+1|}{p} .
$$

Proof. (i) Provided that $-\Delta_{p}\left(r_{+}^{\alpha}\right) \geq 0$ and $c>0$, we set

$$
X:=-\alpha r_{+}^{\beta+1} \nabla r_{+}, f_{X}:=c r_{+}^{\beta} .
$$

Clearly, $f_{X} \in L_{\text {loc }}^{1}(\Omega)$. The Hölder inequality together with Condition (1) implies $r_{+}^{\beta+1} \in L_{\text {loc }}^{1}(\Omega)$ and hence, $X \in L_{\text {loc }}^{1}(T \Omega)$. Moreover, $F^{p}(X) / f_{X}^{p-1} \in L_{\mathrm{loc}}^{1}(\Omega)$ because $r_{+}^{\beta+p} \in L_{\mathrm{loc}}^{1}(\Omega)$.

Given $\epsilon>0$ and $u \in C_{0}^{1}(\Omega)$ with $u \geq 0$, set $r_{\epsilon}:=\epsilon+r_{+}$and $v:=r_{\epsilon}^{-\frac{c}{a}} u$. Since $-\Delta_{p}\left(r_{+}^{\alpha}\right) \geq 0$ and $r_{+}^{(\alpha-1)(p-1)} \in L_{\text {loc }}^{1}(\Omega)$, we have $\int_{\Omega}\left\langle F^{p-2}\left(\nabla r_{+}^{\alpha}\right) \nabla r_{+}^{\alpha}, d v\right\rangle d \mathfrak{m} \geq 0$, that is,

$$
c \alpha^{p-2} \int_{\Omega} r_{+}^{(\alpha-1)(p-1)} u r_{\epsilon}^{-\frac{c}{a}-1} d \mathfrak{m} \leq \alpha^{p-1} \int_{\Omega} r_{+}^{(\alpha-1)(p-1)} r_{\epsilon}^{-\frac{c}{a}}\left\langle\nabla r_{+}, d u\right\rangle d \mathfrak{m} .
$$

Since $r_{+}^{(\alpha-1)(p-1)} r_{\epsilon}^{-\frac{c}{a}-1} \leq r_{+}^{\beta} \in L_{\mathrm{loc}}^{1}(\Omega)$ and $r_{+}^{(\alpha-1)(p-1)} r_{\epsilon}^{-\frac{c}{a}} \leq r_{+}^{\beta+1} \in L_{\mathrm{loc}}^{1}(\Omega)$, the Lebesgue's dominated convergence theorem together with (3.3) yields

$$
\int_{\Omega} c r_{+}^{\beta} u d \mathfrak{m} \leq \int_{\Omega} \alpha r_{+}^{\beta+1}\left\langle\nabla r_{+}, d u\right\rangle d \mathfrak{m}
$$

that is, $f_{X} \leq \operatorname{div} X$ in the weak sense. Now (3.1) follows from Theorem 3.1 (1).

In the case when $-\Delta_{p}\left(r_{+}^{\alpha}\right) \leq 0$ and $c<0$, set $X:=\alpha r_{+}^{\beta+1} \nabla r_{+}, f_{X}:=-c r_{+}^{\beta}$ and $v:=r_{+}^{-c / \alpha} u$. Then (3.1) follows from a similar argument and Theorem 3.1 (2). 
(ii) In order to show (3.2), one set

$$
\begin{cases}X:=-\alpha r^{\beta+1} \nabla\left(-r_{-}\right), f_{X}:=c r_{-}^{\beta}, v:=r_{-}^{\frac{c}{a}} u, & \text { if } c>0 \text { and }-\Delta_{p}\left(r_{-}^{-\alpha}\right) \geq 0, \\ X:=\alpha r^{\beta+1} \nabla\left(-r_{-}\right), f_{X}:=-c r_{-}^{\beta}, v:=\left(r_{-}+\epsilon\right)^{\frac{c}{a}} u, & \text { if } c<0 \text { and }-\Delta_{p}\left(r_{-}^{-\alpha}\right) \leq 0 .\end{cases}
$$

Then the proof follows in a similar manner and hence, we omit it.

We introduce the following space to investigate the sharpness of constants of Hardy inequalities.

Definition 3.5. Let $(M, o, F, d \mathfrak{m})$ be an $n$-dimensional complete reversible PFMMM and let $\Omega \subset M$ be a natural domain. Given $p>1$ and $\beta \in \mathbb{R}$, suppose $o \notin \Omega$ if $\beta \leq-n$. Denote by $D^{1, p}\left(\Omega, r^{\beta+p}\right)$ the closure of $C_{0}^{\infty}(\Omega)$ with respect to the norm

$$
\|u\|_{D}:=\left(\int_{\Omega} r^{\beta}|u|^{p} d \mathfrak{m}+\int_{\Omega} r^{p+\beta} F^{* p}(d u) d \mathfrak{m}\right)^{\frac{1}{p}},
$$

where $r(x):=d_{F}(o, x)$.

Lemma 3.2 implies that both $\|\cdot\|_{D}$ and $D^{1, p}\left(\Omega, r^{\beta+p}\right)$ are well-defined. The following lemma provides the extremal functions for some Hardy inequalities.

Lemma 3.6. Let $(M, o, F, d \mathfrak{m})$ be a complete reversible PFMMM with $\mathbf{S}_{o}^{+} \geq 0$ and either $\mathbf{R i c} \geq 0$ or Ric $_{\infty} \geq 0$. Suppose that $p, \beta \in \mathbb{R}$ and $\Omega$ satisfy one of the following conditions:

(1) $\beta>-n, p>1$ and $\Omega:=M$ is noncompact; $\quad$ (2) $\beta<-n, p>\max \{1,-n-\beta\}$ and $\Omega:=M \backslash\{o\}$. For any $\delta \in(0,1)$ and any $s \in\left(0, \min \left\{1, \mathfrak{i}_{o} / 2\right\}\right)$, set $c(\delta):=|n+\beta| / p+\delta / p$ and

$$
v(x):=\left\{\begin{array}{l}
\left(\frac{r(x)}{s}\right)^{c(\delta)}, \text { if } x \in B_{o}(s), \\
\left(\frac{r(x)}{s}\right)^{-c(\delta / 2)}, \quad \text { if } x \in M \backslash B_{o}(s) .
\end{array}\right.
$$

Thus, $v \in D^{1, p}\left(\Omega, r^{p+\beta}\right)$. In particular,

$$
c^{p}(\delta) \int_{\Omega} r^{\beta}|v|^{p} d \mathfrak{m}>\int_{\Omega} r^{\beta+p} F^{* p}(d v) d \mathfrak{m} .
$$

Proof. For any $\epsilon \in(0,1)$, set $v_{\epsilon}:=\max \{v-\epsilon, 0\}$. Lemma A.1 in Appendix A implies $v_{\epsilon} \in D^{1, p}\left(\Omega, r^{p+\beta}\right)$.

Let $(t, y)$ be the polar coordinate system around $o$. The curvature assumption together with (2.9) and Lemma A.2 implies

$$
\hat{\sigma}_{o}(t, y) \leq e^{-\tau(y)} t^{n-1} \text {, for any } y \in S_{o} M, 0<t<i_{y} .
$$

Firstly, we show that $\|v\|_{D}$ is finite. In fact, since $p c(\delta)+\beta+n>0$ and $p c(\delta)-(\beta+n)>0,(2.7)$ together with (3.6) furnishes

$$
\begin{gathered}
\int_{M} r^{\beta}|v|^{p} d \mathfrak{m}=\int_{B_{o}(s)}\left(\frac{r}{s}\right)^{p c(\delta)} r^{\beta} d \mathfrak{m}+\int_{M \backslash B_{o}(s)}\left(\frac{r}{s}\right)^{-p c(\delta / 2)} r^{\beta} d \mathfrak{m} \\
\leq s^{\beta+n} \int_{S_{o} M} e^{-\tau(y)} d \nu_{o}(y)\left[\frac{1}{p c(\delta)+\beta+n}+\frac{1}{p c(\delta / 2)-(\beta+n)}\right]<+\infty .
\end{gathered}
$$

On the other hand, a direct calculation together with (3.7) yields

$$
\begin{aligned}
& \int_{M} F^{* p}(d v) r^{p+\beta} d \mathfrak{m}=\int_{B_{o}(s)} F^{* p}(d v) r^{p+\beta} d \mathfrak{m}+\int_{M \backslash B_{o}(s)} F^{* p}(d v) r^{p+\beta} d \mathfrak{m} \\
= & c^{p}(\delta) \int_{B_{o}(s)} r^{\beta}|v|^{p} d \mathfrak{m}+c^{p}(\delta / 2) \int_{M \backslash B_{o}(s)} r^{\beta}|v|^{p} d \mathfrak{m}<+\infty .
\end{aligned}
$$

Then the finiteness of $\|v\|_{D}$ follows. Moreover, since $c^{p}(\delta)>c^{p}(\delta / 2)>0$, (3.5) follows from (3.8) immediately. 
Secondly, we prove $\left\|v_{\epsilon}-v\right\|_{D} \rightarrow$ as $\epsilon \rightarrow 0^{+}$. Choose a small $\epsilon \in(0,1)$ such that $\mathfrak{i}_{o}>s \epsilon^{1 / c(\delta)}$. Due to the positivity of $r^{\beta+n-1},(3.6)$ yields

$$
\begin{aligned}
& \int_{B_{o}\left(s \epsilon^{-1 / c(\delta / 2)}\right) \backslash B_{o}\left(s \epsilon^{1 / c(\delta)}\right)} \epsilon^{p} r^{\beta} d \mathfrak{m} \leq \epsilon^{p} \int_{S_{o} M} e^{-\tau(y)} d \nu_{o}(y) \int_{s \epsilon^{1 / c(\delta)}}^{\min \left\{i_{y}, s \epsilon^{-1 / c(\delta / 2)}\right\}} r^{\beta+n-1} d r \\
\leq & \frac{s^{\beta+n}}{\beta+n}\left[\epsilon^{\frac{p c(\delta / 2)-(\beta+n)}{c(\delta / 2)}}-\epsilon^{\frac{p c(\delta)+\beta+n}{c(\delta)}}\right] \int_{S_{o} M} e^{-\tau(y)} d \nu_{o}(y) \rightarrow 0, \text { as } \epsilon \rightarrow 0^{+},
\end{aligned}
$$

which together with $\|v\|_{D}<\infty$ implies

$$
\begin{aligned}
\left\|v_{\epsilon}-v\right\|_{D}^{p}= & \int_{B_{o}\left(s \epsilon^{1 / c(\delta)}\right)}+\int_{M \backslash B_{o}\left(s \epsilon^{-1 / c(\delta / 2)}\right)}|v|^{p} r^{\beta} d \mathfrak{m}+\int_{B_{o}\left(s \epsilon^{-1 / c(\delta / 2)}\right) \backslash B_{o}\left(s \epsilon^{1 / c(\delta)}\right)} \epsilon^{p} r^{\beta} d \mathfrak{m} \\
& +\int_{B_{o}\left(s \epsilon^{1 / c(\delta)}\right)}+\int_{M \backslash B_{o}\left(s \epsilon^{-1 / c(\delta / 2)}\right)} F^{* p}(d v) r^{p+\beta} d \mathfrak{m} \rightarrow 0, \text { as } \epsilon \rightarrow 0^{+} .
\end{aligned}
$$

Now $v \in D^{1, p}\left(\Omega, r^{\beta+p}\right)$ follows from $v_{\epsilon} \in D^{1, p}\left(\Omega, r^{p+\beta}\right)$.

3.2. Finsler manifolds with non-positive flag curvature. In this subsection, we study the Hardy inequalities on FMMMs with non-positive flag curvature. To begin with, we review the Laplacian comparison theorems concerned with the flag curvature.

Lemma 3.7 (cf. $[35,40])$. Let $(M, o, F, d \mathfrak{m})$ be an n-dimensional forward complete PFMMM with $\mathbf{K} \leq 0$. Then the following inequalities hold a.e. on $M$ :

$$
\begin{cases}\Delta r_{+} \geq \frac{n-1}{r_{+}}, & \text {if } \mathbf{S}_{o}^{+} \leq 0, \\ -\Delta\left(-r_{-}\right) \geq \frac{n-1}{r_{-}}, & \text {if } \mathbf{S}_{o}^{-} \geq 0 .\end{cases}
$$

Sketch of the proof. A standard argument (see Shen [35] or Wu and Xin [40]) furnishes $\Delta r_{+} \geq \frac{n-1}{r_{+}}$. In order to show $-\Delta\left(-r_{-}\right) \geq \frac{n-1}{r_{-}}$, consider the RFMMM $(M, \overleftarrow{F}, d \mathfrak{m})$. Thus, (2.10) together with Remark 1 yields $\overleftarrow{\mathbf{K}} \leq 0, \overleftarrow{\mathbf{S}}+\leq 0$ and therefore, the same argument implies $\overleftarrow{\Delta}\left(\overleftarrow{r_{+}}\right) \geq \frac{n-1}{\overleftarrow{r_{+}}}$. We conclude the proof by $\overleftarrow{\Delta}\left(\overleftarrow{r_{+}}\right)=-\Delta\left(-r_{-}\right)$and $\overleftarrow{r_{+}}=r_{-}$

Theorem 3.8. Let $(M, o, F, d \mathfrak{m})$ be an $n$-dimensional forward complete PFMMM with $\mathbf{K} \leq 0$ and $\mathbf{S}_{o}^{-} \geq 0$. Let $\Omega$ be a natural domain with $o \in \Omega$. Given $p, \beta \in \mathbb{R}$ with $p \in(1, n)$ and $\beta>-n$, we have

$$
\int_{\Omega} r_{-}^{\beta+p} \max \left\{F^{* p}( \pm d u)\right\} d \mathfrak{m} \geq\left(\frac{n+\beta}{p}\right)^{p} \int_{\Omega} r_{-}^{\beta}|u|^{p} d \mathfrak{m}, \forall u \in C_{0}^{\infty}(\Omega),
$$

In particular, the constant $\left(\frac{n+\beta}{p}\right)^{p}$ is sharp if $\lambda_{F}(\Omega)=1$.

Proof. Let $\alpha=(n-p) /(p-1)$ and $c=\alpha[(\alpha+1)(p-1)+\beta+1]>0$. A direct calculation together with (3.9) yields

$$
-c \Delta_{p}\left(r_{-}^{-\alpha}\right)=c \alpha^{p-1} r_{-}^{-(\alpha+1)(p-1)-1}\left[-(\alpha+1)(p-1)+r_{-}\left(-\Delta\left(-r_{-}\right)\right)\right] \geq 0 .
$$

And Lemma 3.2 yields $r_{-}^{(-\alpha-1)(p-1)}, r_{-}^{\beta}, r_{-}^{\beta+p} \in L_{\text {loc }}^{1}(\Omega)$. Thus, Lemma 3.4 (ii) furnishes (3.10) immediately.

In the sequel, we show that the constant $\vartheta^{p}:=((n+\beta) / p)^{p}$ is sharp if $\left.F\right|_{\Omega}$ is reversible. Set

$$
\mathfrak{C}_{\beta, p}(\Omega):=\inf _{u \in C_{0}^{\infty}(\Omega) \backslash\{0\}} \frac{\int_{\Omega} r^{\beta+p} F^{* p}(d u) d \mathfrak{m}}{\int_{\Omega} r^{\beta}|u|^{p} d \mathfrak{m}} .
$$

Thus, (3.10) furnishes $\mathfrak{C}_{\beta, p}(\Omega) \geq \vartheta^{p}$. On the other hand, choose $R \in\left(0, \mathfrak{i}_{o}\right)$ such that $B_{o}(R) \subset \subset \Omega$. For any $\epsilon \in(0, R / 4)$, define $u_{\epsilon}(x):=\max \{\epsilon, r(x)\}^{-\vartheta}$ and choose a cut-off function $\phi \in C_{0}^{\infty}(\Omega)$ such that

$$
\phi= \begin{cases}1, & x \in B_{o}(R / 2), \\ 0, & x \notin B_{o}(R) .\end{cases}
$$


Set $v:=\phi u_{\epsilon}$. Lemma A.1 implies $v \in D^{1, p}\left(\Omega, r^{\beta+p}\right)$. And a direct calculation yields

$$
\int_{\Omega} r^{\beta+p} F^{* p}(d v) d \mathfrak{m}=\vartheta^{p} \int_{B_{o}(R / 2) \backslash B_{o}(\epsilon)} r^{-n} d \mathfrak{m}+\int_{B_{o}(R) \backslash B_{o}(R / 2)} r^{\beta+p} F^{* p}\left(d\left(\phi r^{-\vartheta}\right)\right) d \mathfrak{m} .
$$

On the other hand, Lemma 3.2 implies

$$
\int_{\Omega} r^{\beta}|v|^{p} d \mathfrak{m} \geq \int_{B_{o}(R / 2) \backslash B_{o}(\epsilon)} r^{\beta}|v|^{p} d \mathfrak{m}=\int_{B_{o}(R / 2) \backslash B_{o}(\epsilon)} r^{-n} d \mathfrak{m} \rightarrow+\infty, \quad \text { as } \epsilon \rightarrow 0^{+} .
$$

Therefore, the above inequalities furnish

$$
\mathfrak{C}_{\beta, p}(\Omega) \leq \frac{\int_{\Omega} r^{\beta+p} F^{* p}(d v) d \mathfrak{m}}{\int_{\Omega} r^{\beta}|v|^{p} d \mathfrak{m}} \rightarrow \vartheta^{p}, \text { as } \epsilon \rightarrow 0^{+},
$$

which concludes the proof.

Remark 2. Let $(M, F, d \mathfrak{m})$ be an $n$-dimensional forward complete FMMM with $\mathbf{K} \leq 0$ and $\mathbf{S} \geq 0$ and let $\Omega$ be a natural domain. The same argument as above shows that (3.10) remains valid even if $o \notin \Omega$.

Proof of Theorem 1.1. Theorem 1.1 is a direct consequence of Theorem 3.8.

Recently, the $L^{p}$-Hardy inequalities on a reversible Minkowski space endowed with the Lebesgue measure have been investigated in Mercaldo, Sano and Takahshi [25] by different methods. The following example follows from [25, Theorem 1.1, (2.2), Theorem 6.4], which can also be deduced from Theorem 3.8 and Remark 2.

Example 3. Let $\left(\mathbb{R}^{n}, F, d x\right)$ be an $n$-dimensional reversible Minkowski space endowed with the Lebesgue measure. Let $\Omega$ be a domain in $\mathbb{R}^{n}$ and let $r(x):=d_{F}(\mathbf{0}, x)=F(x)$. For $1<p<n$, one has

$$
\left(\frac{n-p}{p}\right)^{p} \int_{\Omega} \frac{|u(x)|^{p}}{r^{p}(x)} d x \leq \int_{\Omega} F^{p}(\nabla u) d x, \forall u \in C_{0}^{\infty}(\Omega) .
$$

Moreover, if $\mathbf{0} \in \Omega$, then $\left(\frac{n-p}{p}\right)^{p}$ is sharp (but not attained).

Theorem 3.9. Let $(M, o, F, d \mathfrak{m})$ be an $n$-dimensional forward complete PFMMM with $\mathbf{K} \leq 0$ and $\mathbf{S}_{o}^{+} \leq 0$. Given any $p, \beta \in \mathbb{R}$ with $p>n>-\beta$, we have

$$
\int_{M} r_{+}^{\beta+p} \max \left\{F^{* p}( \pm d u)\right\} d \mathfrak{m} \geq\left(\frac{n+\beta}{p}\right)^{p} \int_{M} r_{+}^{\beta}|u|^{p} d \mathfrak{m}, \forall u \in C_{0}^{\infty}(M) .
$$

In particular, the constant $\left(\frac{n+\beta}{p}\right)^{p}$ is sharp if $\lambda_{F}=1, \mathfrak{i}_{o}=+\infty, \mathbf{K}=0$ and $\mathbf{S}_{o}^{+}=0$.

Proof. Let $\alpha:=(p-n) /(p-1)$ and $c:=\alpha[(\alpha-1)(p-1)-\beta-1]<0$. A direct calculation together with (3.9) yields

$$
-c \Delta_{p}\left(r_{+}^{\alpha}\right)=-c \alpha^{p-1} r_{+}^{(\alpha-1)(p-1)-1}\left[(\alpha-1)(p-1)+r_{+} \Delta r_{+}\right] \geq 0 .
$$

And Lemma 3.2 implies $r_{+}^{(\alpha-1)(p-1)}, r^{\beta}, r^{\beta+p} \in L_{\text {loc }}^{1}(M)$. Then (3.11) follows from Lemma 3.4 (i) directly.

It remains to show that $\vartheta^{p}:=((n+\beta) / p)^{p}$ is sharp if $\lambda_{F}=1, \mathfrak{i}_{o}=+\infty, \mathbf{K}=0$ and $\mathbf{S}_{o}^{+}=0$. Note that $M$ is noncompact in this case. Now set

$$
\mathfrak{C}_{\beta, p}(M):=\inf _{u \in C_{0}^{\infty}(M) \backslash\{0\}} \frac{\int_{M} r^{\beta+p} F^{* p}(d u) d \mathfrak{m}}{\int_{M} r^{\beta}|u|^{p} d \mathfrak{m}} .
$$

Thus, (3.11) implies $\mathfrak{C}_{\beta, p}(M) \geq \vartheta^{p}$. On the other hand, due to Ric $=0$, Lemma 3.6 furnishes

$$
\mathfrak{C}_{\beta, p}(M) \leq \frac{\int_{M} r^{\beta+p} F^{* p}(d v) d \mathfrak{m}}{\int_{M} r^{\beta}|v|^{p} d \mathfrak{m}}<c^{p}(\delta) \rightarrow \vartheta^{p}, \text { as } \delta \rightarrow 0^{+},
$$

where $v$ is defined as in Lemma 3.6. This concludes the proof.

Proof of Theorem 1.2. Since the injectivity radius of every point in a Cartan-Hadamard manifold is infinite (cf. Bao et al. [5]), Theorem 1.2 follows from Theorem 3.9 immediately. 
A reversible Minkowski space is a linear space equipped with a reversible Minkowski norm. According to Bao et al. [5] and Shen [34,35], every reversible Minkowski space endowed with the Lebesgue measure is a Cartan-Hadamard measure manifold with $\lambda_{F}=1, \mathbf{K}=0$ and $\mathbf{S}=0$. Hence, the inequality (3.11) is optimal on such spaces. There are various reversible Minkowski norms on $\mathbb{R}^{n}$. Now we recall two types of them.

Example 4. 1. $(\alpha, \beta)$-metrics on $\mathbb{R}^{n}$. See Chern and Shen [9]. Let $\phi(s)$ be an arbitrary $C^{\infty}$ even function on some symmetric open interval $I=\left(-b_{0}, b_{0}\right)$ with

$$
\phi(s)>0,\left(\phi(s)-s \phi^{\prime}(s)\right)+\left(b^{2}-s^{2}\right) \phi^{\prime \prime}(s)>0,
$$

where $s$ and $b$ are arbitrary numbers with $|s|<b<b_{0}$. Then for any constant 1 -form $\beta(y)=b_{i} y^{i}$ on $\mathbb{R}^{n}$ with $\|\beta\|<b_{0}$, the following metric

$$
F(y):=\|y\| \phi\left(\frac{\beta(y)}{\|y\|}\right), \forall y \in T \mathbb{R}^{n}
$$

is a reversible Minkowski norm on $\mathbb{R}^{n}$, where $\|\cdot\|$ denotes the Euclidean norm.

2. Fourth Root metrics on $\mathbb{R}^{n}$. See Li and Shen [22]. Let $A(y):=a_{i j k l} y^{i} y^{j} y^{k} y^{l}$. Suppose $\left(2 A A_{i j}-A_{i} A_{j}\right)$ is positive definite, where $A_{i}:=\frac{\partial A}{\partial y^{i}}$ and $A_{i j}:=\frac{\partial^{2} A}{\partial y^{i} \partial y^{j}}$. Then $F(y):=A^{\frac{1}{4}}$ is a reversible Minkowski norm on $\mathbb{R}^{n}$.

Given a forward complete Finsler manifold $(M, F)$, the topology induced by backward balls is the same as the original one (cf. Bao et al. [5, p.155]). Denote by $\overleftarrow{\mathfrak{i}_{o}}$ (resp., $\mathfrak{i}_{o}$ ) the injectivity radius of $o$ with respect to $\left(M, \overleftarrow{F}\right.$ ) (resp., $(M, F)$ ). A standard argument shows both $\overleftarrow{\mathfrak{i}_{o}}, \mathfrak{i}_{o}$ are positive (see Bao et al. [5, Theorem 6.3.1] and Yuan et al. [43, Proposition 3.2] for example). However, $\overleftarrow{\mathfrak{i}_{o}}$ may not coincide with $\mathfrak{i}_{o}$ if $\lambda_{F} \neq 1$. For instance, for the Funk manifold in Example $1, \mathfrak{i}_{0}=+\infty$ while $\overleftarrow{\mathfrak{i}_{0}}=\log 2$. Now we have the following result.

Theorem 3.10. Let $(M, o, F, d \mathfrak{m})$ be an $n$-dimensional forward complete PFMMM with $\mathbf{K} \leq 0$ and $\mathbf{S}_{o}^{-} \geq 0$. For any $R, p, \beta \in \mathbb{R}$ with $R \in\left(0, \overleftarrow{\mathfrak{i}_{o}}\right), p \in(1, n]$ and $\beta<-1$, we have

$$
\int_{B_{o}^{-}(R)}\left[\log \left(\frac{R}{r_{-}}\right)\right]^{p+\beta} \max \left\{F^{* p}( \pm d u)\right\} d \mathfrak{m} \geq\left(\frac{|\beta+1|}{p}\right)^{p} \int_{B_{o}^{-}(R)}\left[\log \left(\frac{R}{r_{-}}\right)\right]^{\beta} \frac{|u|^{p}}{r_{-}^{p}} d \mathfrak{m}
$$

for any $u \in C_{0}^{\infty}\left(B_{o}^{-}(R)\right)$. In particular, the constant $\left(\frac{|\beta+1|}{p}\right)^{p}$ is sharp if $\lambda_{F}\left(B_{o}^{-}(R)\right)=1$.

Proof. The proof is divided into three steps.

Step 1. Let $\rho:=\log \left(\frac{R}{r_{-}}\right)$. Obviously, $\rho^{\beta}$ can be viewed as a continuous function on $B_{o}^{-}(R)$ by setting $\rho^{\beta}(o)=0$. Now we claim

$$
F^{(p-1)}(\nabla \rho), \rho^{\beta} F^{p}(\nabla \rho), \rho^{\beta+p}, \rho^{\beta+1} F^{p-1}(\nabla \rho) \in L_{\mathrm{loc}}^{1}\left(B_{o}^{-}(R)\right) .
$$

(1) Since $p \leq n$, Lemma 3.2 implies $F^{(p-1)}(\nabla \rho)=1 / r_{-}^{p-1} \in L_{\text {loc }}^{1}\left(B_{o}^{-}(R)\right)$.

(2) Let $(\mathfrak{t}, \mathfrak{y})$ be the polar coordinate system around $o$ in the $\operatorname{RFMMM}(M, \overleftarrow{F}, d \mathfrak{m})$. Thus, $\mathfrak{t}=r_{-}(x)$ According to $(2.8)$, there exists an $\epsilon \in(0, \min \{1, R / 2\})$ such that for any $\mathfrak{y} \in \overleftarrow{S_{o} M}$,

$$
0<\overleftarrow{\hat{\sigma}_{o}}(\mathfrak{t}, \mathfrak{y})<2 e^{-\overleftarrow{\tau}(\mathfrak{y})} \mathfrak{t}^{n-1}, 0<\mathfrak{t}<\epsilon
$$


Here, we use $\overleftarrow{*}$ to denote the corresponding geometric quantity $*$ in $(M, \overleftarrow{F})$. Since $n-1-p \geq-1$ and $\beta<-1,(2.7)$ together with (2.10) and (3.14) yields

$$
\begin{aligned}
\int_{B_{o}^{-}(\epsilon)} \rho^{\beta} F^{p}(\nabla \rho) d \mathfrak{m} & =\int_{\overleftarrow{B_{o}^{+}(\epsilon)}} \rho^{\beta} F^{p}(\nabla \rho) d \mathfrak{m}=\int_{\overleftarrow{S_{o} M}} d \overleftarrow{\nu_{o}}(\mathfrak{y}) \int_{0}^{\epsilon}\left[\log \left(\frac{R}{\mathfrak{t}}\right)\right]^{\beta} \frac{1}{\mathfrak{t}^{p}} \overleftarrow{\sigma_{o}}(\mathfrak{t}, \mathfrak{y}) d \mathfrak{t} \\
& \leq 2 \int_{\overleftarrow{S_{o} M}} e^{-\overleftarrow{\tau}(\mathfrak{y})} d \overleftarrow{\nu_{o}}(\mathfrak{y}) \int_{0}^{\epsilon}\left[\log \left(\frac{R}{\mathfrak{t}}\right)\right]^{\beta} \mathfrak{t}^{n-1-p} d \mathfrak{t} \\
& \leq \frac{2}{|1+\beta|}\left[\log \left(\frac{R}{\epsilon}\right)\right]^{\beta+1} \int_{\overleftarrow{S_{o} M}} e^{-\overleftarrow{\tau}(\mathfrak{y})} d \overleftarrow{\nu_{o}}(\mathfrak{y})<+\infty,
\end{aligned}
$$

which implies $\rho^{\beta} F^{p}(\nabla \rho) \in L_{\text {loc }}^{1}\left(B_{o}^{-}(R)\right)$.

(3) Since $\beta+p<n-1$, we choose a small $\epsilon \in(0, \min \{1, R / 2\})$ such that

$$
\frac{(\log s)^{\beta+p}}{s^{n-1}} \leq 1, \text { for } s \in(R / \epsilon,+\infty) .
$$

A calculation similar to (3.15) then yields

$$
\begin{aligned}
\int_{B_{o}^{-}(\epsilon)} \rho^{\beta+p} d \mathfrak{m} & \leq 2 \int_{\overleftarrow{S_{o} M}} e^{-\overleftarrow{\tau}(\mathfrak{y})} d \overleftarrow{\nu_{o}}(\mathfrak{y}) \int_{0}^{\epsilon}\left[\log \left(\frac{R}{\mathfrak{t}}\right)\right]^{\beta+p} \mathfrak{t}^{n-1} d \mathfrak{t} \\
& \leq 2 R^{n-1} \epsilon \int_{\overleftarrow{S_{o} M}} e^{-\overleftarrow{\tau}(\mathfrak{y})} d \overleftarrow{\nu_{o}}(\mathfrak{y})<+\infty
\end{aligned}
$$

which implies $\rho^{\beta+p} \in L_{\mathrm{loc}}^{1}\left(B_{o}^{-}(R)\right)$.

(4) The Hölder inequality together with (2) and (3) furnishes $\rho^{\beta+1} F^{p-1}(\nabla \rho) \in L_{\text {loc }}^{1}\left(B_{o}^{-}(R)\right)$.

Therefore, (3.13) follows as claimed.

Step 2. In this step, we prove (3.12). In order to do this, set $X:=-\rho^{\beta+1} F^{p-2}(\nabla \rho) \nabla \rho$ and $f_{X}:=$ $c \rho^{\beta} F^{p}(\nabla \rho)$, where $c:=-\beta-1=|\beta+1|>0$. Then (3.13) indicates $X \in L_{\text {loc }}^{1}\left(T B_{o}^{-}(R)\right), f_{X} \in L_{\text {loc }}^{1}\left(B_{o}^{-}(R)\right)$ and $F^{p}(X) / f_{X}^{p-1} \in L_{\mathrm{loc}}^{1}\left(B_{o}^{-}(R)\right)$.

If $f_{X} \leq \operatorname{div} X$ in the weak sense, then (3.12) would follow from Theorem 3.1 (1) immediately. Therefore, it suffices to show that $f_{X} \leq \operatorname{div} X$ in the weak sense, i.e.,

$$
\int_{B_{o}^{-}(R)} \rho^{\beta+1} F^{p-2}(\nabla \rho)\langle\nabla \rho, d u\rangle d \mathfrak{m} \geq c \int_{B_{o}^{-}(R)} \rho^{\beta} F^{p}(\nabla \rho) u d \mathfrak{m}, \forall u \in C_{0}^{\infty}\left(B_{o}^{-}(R)\right) \text { with } u \geq 0 .
$$

We proceed as follows. Set $v:=\rho^{-c} u$. Thus, (3.13) together with (2.2) yields

$$
\begin{aligned}
& \left\langle F^{p-2}(\nabla \rho) \nabla \rho, u \rho^{-c-1} d \rho\right\rangle=F^{p}(\nabla \rho) \rho^{\beta} u \in L^{1}\left(B_{o}^{-}(R)\right), \\
& \left|\left\langle F^{p-2}(\nabla \rho) \nabla \rho, \rho^{-c} d u\right\rangle\right| \leq \lambda_{F}(\operatorname{supp}(u)) \rho^{\beta+1} F^{p-1}(\nabla \rho) F^{*}(d u) \in L^{1}\left(B_{o}^{-}(R)\right),
\end{aligned}
$$

which imply

$$
\left\langle F^{p-2}(\nabla \rho) \nabla \rho, d v\right\rangle=-c\left\langle F^{p-2}(\nabla \rho) \nabla \rho, u \rho^{-c-1} d \rho\right\rangle+\left\langle F^{p-2}(\nabla \rho) \nabla \rho, \rho^{-c} d u\right\rangle \in L^{1}\left(B_{o}^{-}(R)\right) .
$$

Choose a small $\delta \in(0, \epsilon)$, where $\epsilon$ is defined as in (3.14). Thus, (3.17) furnishes

$$
\lim _{\delta \rightarrow 0^{+}} \int_{\frac{B_{o}^{-}(\delta)}{}}\left\langle F^{p-2}(\nabla \rho) \nabla \rho, d v\right\rangle d \mathfrak{m}=0 .
$$

On the other hand, (3.9) yields $-\Delta_{p} \rho=r_{-}^{-p}\left[(1-p)+r_{-}\left(-\Delta\left(-r_{-}\right)\right)\right] \geq \frac{(n-p)}{r_{-}^{p}} \geq 0$, which together with (2.6) yields

$$
\begin{aligned}
0 & \leq-\int_{B_{o}^{-}(R) \backslash \overline{B_{o}^{-}(\delta)}} v \Delta_{p} \rho d \mathfrak{m}=-\int_{B_{o}^{-}(R) \backslash \overline{B_{o}^{-}(\delta)}}\left[\operatorname{div}\left(v F^{p-2}(\nabla \rho) \nabla \rho\right)-\left\langle F^{p-2}(\nabla \rho) \nabla \rho, d v\right\rangle\right] d \mathfrak{m} \\
& =-\int_{\partial B_{o}^{-}(\delta)} g_{\mathbf{n}_{-}}\left(\mathbf{n}_{-}, F^{p-2}(\nabla \rho) \nabla \rho\right) v d A+\int_{B_{o}^{-}(R) \backslash \overline{B_{o}^{-}(\delta)}}\left\langle F^{p-2}(\nabla \rho) \nabla \rho, d v\right\rangle d \mathfrak{m},
\end{aligned}
$$

where $\mathbf{n}_{-}$is the unit inward normal vector field on $\partial B_{o}^{-}(\delta)$ and $d A$ is the induced measure on $\partial B_{o}^{-}(\delta)$. 
Let $(\mathfrak{t}, \mathfrak{y})$ be the polar coordinates as in Step 1. Thus, $d A=\overleftarrow{\sigma_{o}}(\delta, \mathfrak{y}) d \overleftarrow{\nu_{o}}(\mathfrak{y})$. Since $\delta \in(0, \epsilon),(2.1)$ together with (3.14) yields

$$
\begin{aligned}
& \left|\int_{\partial B_{o}^{-}(\delta)} g_{\mathbf{n}_{-}}\left(\mathbf{n}_{-}, F^{p-2}(\nabla \rho) \nabla \rho\right) v d A\right| \leq \lambda_{F}\left(\partial B_{o}^{-}(\delta)\right) \int_{\partial B_{o}^{-}(\delta)} F^{p-1}(\nabla \rho) \rho^{-c} u d A \\
\leq & 2 \lambda_{F}\left(\partial B_{o}^{-}(\delta)\right) \delta^{n-p}\left[\log \left(\frac{R}{\delta}\right)\right]^{-c} \max _{\partial B_{o}^{-}(\delta)} u \int_{\overleftarrow{S_{o} M}} e^{-\overleftarrow{\tau}(\mathfrak{y})} d \overleftarrow{\nu_{o}}(\mathfrak{y}) \rightarrow 0, \text { as } \delta \rightarrow 0^{+} .
\end{aligned}
$$

Now it follows from (3.18)-(3.20) that $\int_{B_{o}^{-}(R)}\left\langle F^{p-2}(\nabla \rho) \nabla \rho, d v\right\rangle d \mathfrak{m} \geq 0$, which together with (3.17) furnishes (3.16).

Step 3. Now we show the constant $\vartheta^{p}:=\left(\frac{|\beta+1|}{p}\right)^{p}$ is sharp if $\lambda_{F}\left(B_{o}^{-}(R)\right)=1$. In this case, $r_{+}(x)=$ $r_{-}(x)=: r(x)$ for all $x \in B_{o}^{-}(R)$ and hence, $B_{o}^{+}(R)=B_{o}^{-}(R)=: B_{o}(R)$ and $\mathfrak{i}_{o} \geq R$. Set

$$
\mathfrak{C}_{\beta, p}\left(B_{o}(R)\right):=\inf _{u \in C_{0}^{\infty}\left(B_{o}(R)\right) \backslash\{0\}} \frac{\int_{B_{o}(R)} \rho^{p+\beta} F^{* p}(d u) d \mathfrak{m}}{\int_{B_{o}(R)} \rho^{\beta} \frac{|u|^{p}}{r^{p}} d \mathfrak{m}} .
$$

Thus, (3.12) implies $\mathfrak{C}_{\beta, p}\left(B_{o}(R)\right) \geq \vartheta^{p}$. For the reverse inequality, set $c(\delta):=\frac{|\beta+1|+\delta}{p}$ for $\delta \in(0,1)$ and define a function $v$ on $B_{o}(R)$ by

$$
v(x):= \begin{cases}\left(\frac{\rho(x)}{s}\right)^{-c(\delta / 2)}, & \text { if } x \in B_{o}(R / 2), \\ \left(\frac{\rho(x)}{s}\right)^{c(\delta)}, & \text { if } x \in B_{o}(R) \backslash B_{o}(R / 2),\end{cases}
$$

where $s:=\log 2>0$.

By (3.12), we define $D^{1, p}\left(B_{o}(R), \rho^{\beta+p}\right)$ as the completion of $C_{0}^{\infty}\left(B_{o}(R)\right)$ with respect to the norm $|u|_{D}:=\left(\int_{B_{o}(R)} \rho^{p+\beta} F^{* p}(d u) d \mathfrak{m}\right)^{\frac{1}{p}}$. If $v \in D^{1, p}\left(B_{o}(R), \rho^{\beta+p}\right)$, then an easy calculation similar to (3.8) would furnish

$$
\mathfrak{C}_{\beta, p}\left(B_{o}(R)\right) \leq \frac{\int_{B_{o}(R)} \rho^{p+\beta} F^{* p}(d v) d \mathfrak{m}}{\int_{B_{o}(R)} \rho^{\beta} \frac{|u|^{p}}{r^{p}} d \mathfrak{m}}<c^{p}(\delta) \rightarrow \vartheta^{p}, \text { as } \delta \rightarrow 0^{+},
$$

which would conclude the proof. Hence, it suffices to show $v \in D^{1, p}\left(B_{o}(R), \rho^{\beta+p}\right)$.

In order to do this, we first prove $|v|_{D}<\infty$. Let $(t, y)$ be the polar coordinate system around $o$ in $(M, F)$. Since $\mathbf{K} \leq 0$ and $\overline{B_{o}(R)}$ is compact (cf. Bao et al. [5, Theorem 6.6.1]), Remark 1 together with (2.10) and $\lambda_{F}\left(B_{o}(R)\right)=1$ yields two constants $k, h \in \mathbb{R}$ with

$$
-k^{2} \leq \mathbf{R i c}(\nabla t) \leq 0,-h^{2} \leq \mathbf{S}(\nabla t) \leq 0, \forall y \in S_{o} M, 0 \leq t \leq R,
$$

which together with (2.9) yields

$$
\hat{\sigma}_{o}(t, y) \leq e^{-\tau(y)+h^{2} t} \mathfrak{s}_{-k^{2}}^{n-1}(t), \forall y \in S_{o} M, 0<t<R \leq \mathfrak{i}_{o}
$$

Now we study $\int_{B_{o}(R)} \rho^{p+\beta} F^{* p}(d v) d \mathfrak{m}$. Let $\epsilon$ be defined as in (3.14). Since $\beta+1<0$ and $n-1-p \geq-1$, (2.7) together with (3.14) then yields

$$
\int_{B_{o}(\epsilon)} \rho^{p+\beta} F^{* p}(d v) d \mathfrak{m} \leq \frac{2 c^{p}(\delta / 2)}{s^{-c(\delta / 2) p}} \int_{S_{o} M} e^{-\tau(y)} d \nu_{o}(y) \int_{0}^{\epsilon}\left[\log \left(\frac{R}{t}\right)\right]^{\beta-c(\delta / 2) p} \frac{1}{t} d t<+\infty .
$$

On the other hand, (2.7) together with (3.21) implies

$$
\begin{aligned}
\int_{B_{o}(R) \backslash B_{o}(R / 2)} \rho^{p+\beta} F^{* p}(d v) d \mathfrak{m} & \leq\left(\frac{2 c(\delta)}{R s^{c(\delta)}}\right)^{p} \int_{S_{o} M} e^{-\tau(y)} d \nu_{o}(y) \int_{R / 2}^{R}\left[\log \left(\frac{R}{t}\right)\right]^{-1+\delta} \mathfrak{s}_{-k^{2}}^{n-1}(t) e^{h^{2} t} d t \\
& \leq \frac{(2 c(\delta))^{p} e^{h^{2} R_{\mathfrak{s}^{n-1}}^{n-1}(R)}}{R^{p-1} s^{c(\delta p)}} \int_{S_{o} M} e^{-\tau(y)} d \nu_{o}(y) \int_{R / 2}^{R}\left[\log \left(\frac{R}{t}\right)\right]^{-1+\delta} \frac{d t}{t}<+\infty
\end{aligned}
$$


which together with (3.22) indicates $\int_{B_{o}(R)} \rho^{p+\beta} F^{* p}(d v) d \mathfrak{m}<\infty$, i.e., $|v|_{D}<\infty$.

Now set $v_{\epsilon}:=\max \{v-\epsilon, 0\}$. By the finiteness of $|v|_{D}$, one can easily check $\left|v_{\epsilon}-v\right|_{D} \rightarrow 0$ as $\epsilon \rightarrow 0^{+}$. On the other hand, a similar argument as in the proof of Lemma A.1 yields $v_{\epsilon} \in D^{1, p}\left(B_{o}(R), \rho^{\beta+p}\right)$. Therefore, $v \in D^{1, p}\left(B_{o}(R), \rho^{\beta+p}\right)$.

Proof of Theorem 1.3. For a reversible Cartan-Hadamard manifold, $\overleftarrow{\mathfrak{i}_{o}}=\mathfrak{i}_{o}=+\infty$. Thus, Theorem 1.3 follows from Theorem 3.10 directly.

3.3. Finsler manifolds with nonnegative Ricci curvature. In this subsection, we consider the Hardy inequalities on FMMMs with nonnegative (weighted) Ricci curvature. We begin by recalling the Laplacian comparison theorems concerned with the (weighted) Ricci curvature.

Lemma 3.11 (cf. $[27,35,40,42])$. Let $(M, o, F, d \mathfrak{m})$ be an $n$-dimensional forward complete PFMMM.

(i) If $\mathbf{R i c}_{N} \geq 0, N \in[n,+\infty)$, then $\Delta r_{+} \leq \frac{N-1}{r_{+}}$and $-\Delta\left(-r_{-}\right) \leq \frac{N-1}{r_{-}}$hold a.e. on $M$.

(ii) Suppose either $\mathbf{R i c}_{\infty} \geq 0$ or $\mathbf{R i c} \geq 0$. Then the following inequalities hold a.e. on $M$ :

$$
\begin{cases}\Delta r_{+} \leq \frac{n-1}{r_{+}}, & \text {if } \mathbf{S}_{o}^{+} \geq 0 \\ -\Delta\left(-r_{-}\right) \leq \frac{n-1}{r_{-}}, & \text {if } \mathbf{S}_{o}^{-} \leq 0\end{cases}
$$

Sketch of the proof. First we consider the case of $r_{+}$. Then (i) is Ohta and Sturm [27, Theorem 5.2] while (ii) follows from the standard Laplacian comparison theorem (cf. Shen [35] or Wu and Xin [40]) and Lemma A.2 (also see Yin [42, Theorem A]). And an argument based on the RFMMM furnishes the results in the case of $r_{-}$.

Now we show Theorem 1.4.

Proof of Theorem 1.4. (1) Clearly, $\Omega:=M \backslash\{o\}$ is an natural domain. Let $\alpha=(p-N) /(p-1)$ and $c:=\alpha[(\alpha-1)(p-1)-\beta-1] \geq 0$. A direct calculation together with Lemma 3.11 (i) furnishes

$$
-c \Delta_{p}\left(r_{+}^{\alpha}\right)=-c \alpha^{p-1} r_{+}^{(\alpha-1)(p-1)-1}\left[(\alpha-1)(p-1)+r_{+} \Delta r_{+}\right] \geq 0 .
$$

Thus, Lemma 3.4 (i) yields (1.4) immediately. In the sequel, we show $\vartheta^{p}:=\left(\frac{|n+\beta|}{p}\right)^{p}$ is $\operatorname{sharp}$ if $\lambda_{F}=1$, $N=n$ and $p+\beta>-n$. In this case, $\mathbf{R i c}_{N} \geq 0$ means $\mathbf{R i c}=\mathbf{R i c}_{n} \geq 0$ and $\mathbf{S}=0$. Then the sharpness follows from the same argument as in the proof of Theorem 3.9.

(2) Let $\alpha=(N-p) /(p-1)$ and $c=\alpha[(\alpha+1)(p-1)+\beta+1]<0$. A direct calculation together with Lemma 3.4 (ii) yields

$$
-c \Delta_{p}\left(r_{-}^{-\alpha}\right)=c \alpha^{p-1} r_{-}^{-(\alpha+1)(p-1)-1}\left[-(\alpha+1)(p-1)+r_{-}\left(-\Delta\left(-r_{-}\right)\right)\right] \geq 0 .
$$

The rest proof is the same as (1) and hence, we omit it.

A similar argument also furnishes the $\mathbf{R i c}_{\infty} / \mathbf{R i c}$ version of Theorem 1.4. We omit the proof.

Theorem 3.12. Let $(M, o, F, d \mathfrak{m})$ be an n-dimensional forward complete PFMMM with either $\mathbf{R i c}_{\infty} \geq 0$ or $\mathbf{R i c} \geq 0$.

(1) Suppose $\mathbf{S}_{o}^{+} \geq 0$. Given $p, \beta \in \mathbb{R}$ with $p>n$ and $\beta<-n$, for any $u \in C_{0}^{\infty}(M \backslash\{o\})$, we have

$$
\int_{M \backslash\{o\}} r_{+}^{\beta+p} \max \left\{F^{* p}( \pm d u)\right\} d \mathfrak{m} \geq\left(\frac{|n+\beta|}{p}\right)^{p} \int_{M \backslash\{o\}} r_{+}^{\beta}|u|^{p} d \mathfrak{m} .
$$

(2) Suppose $\mathbf{S}_{o}^{-} \leq 0$. Given $p, \beta \in \mathbb{R}$ with $1<p<n$ and $\beta<-n$, for any $u \in C_{0}^{\infty}(M \backslash\{o\})$, we have

$$
\int_{M \backslash\{o\}} r_{-}^{\beta+p} \max \left\{F^{* p}( \pm d u)\right\} d \mathfrak{m} \geq\left(\frac{|n+\beta|}{p}\right)^{p} \int_{M \backslash\{o\}} r_{-}^{\beta}|u|^{p} d \mathfrak{m} .
$$

In particular, the constants in (1) and (2) are sharp if $\lambda_{F}=1$ and $p+\beta>-n$.

In the sequel, we present two applications of Theorem 3.12 . 
Definition 3.13. Let $(M, F, d \mathfrak{m})$ be an $n$-dimensional closed reversible FMMM. Given $o \in M$, set $r(x):=$ $d_{F}(o, x)$. Let $\Omega$ be either $M$ or $M \backslash\{o\}$. Given $p \in(1,+\infty)$ and $\beta<-n$ with $p+\beta>-n$, define $W^{1, p}\left(\Omega, r^{\beta+p}\right)$ as the completion of $C_{0}^{\infty}(\Omega)$ under the norm

$$
\|u\|_{p, \beta}:=\left(\int_{\Omega}|u|^{p} r^{p+\beta} d \mathfrak{m}+\int_{\Omega} F^{* p}(d u) r^{p+\beta} d \mathfrak{m}\right)^{\frac{1}{p}} .
$$

By the compactness of $M$, one can easily verify $D^{1, p}\left(M \backslash\{o\}, r^{p+\beta}\right) \subset W^{1, p}\left(M \backslash\{o\}, r^{p+\beta}\right)$, where $D^{1, p}\left(M \backslash\{o\}, r^{p+\beta}\right)$ is defined in Definition 3.5. Moreover, Theorem 3.12 yields the following result.

Theorem 3.14. Let $(M, o, F, d \mathfrak{m})$ be an n-dimensional closed reversible PFMMM with $\mathbf{S}_{o}^{+} \geq 0$ and either $\mathbf{R i c}_{\infty} \geq 0$ or Ric $\geq 0$. Then for any $p \in(1, n) \cup(n,+\infty)$ and $\beta<-n$ with $p+\beta>-n$, we have

$$
D^{1, p}\left(M \backslash\{o\}, r^{p+\beta}\right)=W^{1, p}\left(M \backslash\{o\}, r^{p+\beta}\right) .
$$

Proof. It is enough to show $W^{1, p}\left(M \backslash\{o\}, r^{p+\beta}\right) \subset D^{1, p}\left(M \backslash\{o\}, r^{p+\beta}\right)$. For any $u \in W^{1, p}\left(M \backslash\{o\}, r^{p+\beta}\right)$, there exists a sequence $u_{j} \in C_{0}^{\infty}(M \backslash\{o\})$ converging to $u$ under $\|\cdot\|_{p, \beta}$. Thus, for any $\epsilon>0$, there exists $N>0$ such that for any $j>N,\left\|u_{j}-u\right\|_{p, \beta}<\left(\frac{|n+\beta|}{p}\right) \epsilon$. And Lemma B.1 in Appendix B implies that $u_{j}$ also converges to $u$ pointwise a.e.. Now for $i>N$, Fatou's lemma together with Theorem 3.12 yields

$$
\begin{aligned}
& \left(\int_{M \backslash\{o\}}\left|u_{i}-u\right|^{p} r^{\beta} d \mathfrak{m}\right)^{\frac{1}{p}}=\left(\int_{M \backslash\{o\}} \liminf _{j \rightarrow+\infty}\left|u_{i}-u_{j}\right|^{p} r^{\beta} d \mathfrak{m}\right)^{\frac{1}{p}} \leq \liminf _{j \rightarrow+\infty}\left(\int_{M \backslash\{o\}}\left|u_{i}-u_{j}\right|^{p} r^{\beta} d \mathfrak{m}\right)^{\frac{1}{p}} \\
\leq & \left(\frac{|n+\beta|}{p}\right)^{-1} \liminf _{j \rightarrow+\infty}\left(\int_{M} r^{\beta+p} F^{* p}\left(d u_{i}-d u_{j}\right) d \mathfrak{m}\right)^{\frac{1}{p}} \leq\left(\frac{|n+\beta|}{p}\right)^{-1} \liminf _{j \rightarrow+\infty}\left\|u_{i}-u_{j}\right\|_{p, \beta} \\
\leq & \left(\frac{|n+\beta|}{p}\right)^{-1} \liminf _{j \rightarrow+\infty}\left(\left\|u_{i}-u\right\|_{p, \beta}+\left\|u_{j}-u\right\|_{p, \beta}\right) \leq \epsilon
\end{aligned}
$$

which together with $\left\|u_{i}-u\right\|_{p, \beta} \rightarrow 0$ implies $\left\|u_{i}-u\right\|_{D} \rightarrow 0$ and hence, $u \in D^{1, p}\left(M \backslash\{o\}, r^{r+\beta}\right)$.

By the zero extension, $W^{1, p}\left(M \backslash\{o\}, r^{\beta+p}\right)$ can be viewed as a subset of $W^{1, p}\left(M, r^{\beta+p}\right)$. On the other hand, we have the following result, whose proof is postponed until Appendix B.

Proposition 3.15. Let $(M, F, d \mathfrak{m}), r, p$ and $\beta$ be as in Definition 3.13. If $u \in W^{1, p}\left(M, r^{\beta+p}\right) \cap C(M)$ with $u(o)=0$, then $\left.u\right|_{M \backslash\{o\}} \in W^{1, p}\left(M \backslash\{o\}, r^{\beta+p}\right)$.

The second application of Theorem 3.12 is as follows.

Theorem 3.16. Let $(M, o, F, d \mathfrak{m})$ be an $n$-dimensional closed reversible PFMMM with $\mathbf{R i c}_{\infty} \geq 0$ and $\mathbf{S}_{o}^{+} \geq 0$. Thus, for any $p \in(1, n) \cup(n, \infty)$ and $\beta<-n$ with $p+\beta>-n$, we have

$$
\int_{M} r^{\beta+p} F^{*}(d u)^{p} d \mathfrak{m} \geq\left(\frac{|n+\beta|}{p}\right)^{p} \int_{M} r^{\beta}|u|^{p} d \mathfrak{m}, \forall u \in \mathfrak{C}^{\infty}(M, o),
$$

where $\mathfrak{C}^{\infty}(M, o):=\left\{u \in C^{\infty}(M): u(o)=0\right\}$. In particular, $\left(\frac{|n+\beta|}{p}\right)^{p}$ is sharp with respect to $\mathfrak{C}^{\infty}(M, o)$.

Proof. Given $u \in \mathfrak{C}^{\infty}(M, o)$, Proposition 3.15 together with Theorem 3.14 implies that $\left.u\right|_{M \backslash\{o\}}$ belongs to $D^{1, p}\left(M \backslash\{o\}, r^{p+\beta}\right)$. Hence, there is a sequence $u_{j} \in C_{0}^{\infty}(M \backslash\{o\})$ with $\left\|u_{j}-\left.u\right|_{M \backslash\{o\}}\right\|_{D} \rightarrow 0$, which together with Theorem 3.12 furnishes (3.23).

For the sharpness of the constant, let $v \in D^{1, p}\left(M \backslash\{o\}, r^{p+\beta}\right)$ be defined as in Lemma 3.6. Thus, there exist a sequence $v_{j} \subset C_{0}^{\infty}(M \backslash\{o\})$ with $\left\|v_{j}-v\right\|_{D} \rightarrow 0$. By the zero extension, $v_{j}$ can be viewed as a function in $\mathfrak{C}^{\infty}(M, o)$. Due to this fact, the rest proof is the same as the one of Theorem 3.9.

Remark 3. Theorem 3.16 indicates what kind of function the Hardy inequality remains valid for on a closed manifold. And Theorem 3.16 factually holds for any $u \in W^{1, p}\left(M, r^{p+\beta}\right) \cap C(M)$ with $u(o)=0$.

Proof of Theorem 1.5. According to Example 2, the assumption implies $\mathbf{R i c} \mathbf{c}_{\infty} \geq 0$ and $\mathbf{S}_{o}^{+} \geq 0$. Thus, Theorem 1.5 follows from Theorem 3.16 directly. 


\section{HARDY INEQUALity FOR $p$-SUB/SUPERHARMONIC FUNCTIONS}

4.1. A weighted Hardy inequality. In the section, we study the Hardy inequalities for $p$-sub/superharmonic functions in the Finsler setting. Inspired by D'Ambrosio and Dipierro [13], we have the following result.

Theorem 4.1. Let $(M, F, d \mathfrak{m})$ be a forward complete FMMM and let $\Omega$ be a natural domain in $M$. Given $p>1$ and $\alpha \in \mathbb{R}$, let $\rho \in W_{\text {loc }}^{1, p}(\Omega)$ be a nonnegative function satisfying the following conditions:

(1) $-(p-1-\alpha) \Delta_{p} \rho \geq 0$ in the weak sense;

(2) Additionally suppose $\frac{F^{p}(\nabla \rho)}{\rho^{p-\alpha}}, \rho^{\alpha} \in L_{\text {loc }}^{1}(\Omega)$ if $\alpha>p$.

Then we have the following weighted Hardy inequality

$$
\int_{\Omega} \rho^{\alpha} \max \left\{F^{* p}( \pm d u)\right\} d \mathfrak{m} \geq\left(\frac{|p-1-\alpha|}{p}\right)^{p} \int_{\Omega} \frac{F^{p}(\nabla \rho)}{\rho^{p-\alpha}}|u|^{p} d \mathfrak{m}, \forall u \in C_{0}^{\infty}(\Omega) .
$$

Proof. If $p-\alpha-1=0,(4.1)$ is trivial. So we assume $p-\alpha-1 \neq 0$ in the sequel. Given $\varepsilon \in(0,1)$, set $\rho_{\varepsilon}:=\rho+\varepsilon$ and

$$
X:=-(p-1-\alpha) \frac{F^{p-2}\left(\nabla \rho_{\varepsilon}\right) \nabla \rho_{\varepsilon}}{\rho_{\varepsilon}^{p-1-\alpha}}, f_{X}:=(p-1-\alpha)^{2} \frac{F^{p}\left(\nabla \rho_{\varepsilon}\right)}{\rho_{\varepsilon}^{p-\alpha}} .
$$

The proof is divided into two steps.

Step 1. In this step, we show that (4.1) holds if $f_{X} \leq \operatorname{div} X$ in the weak sense.

In fact, if $p-1-\alpha>0$ (resp., $p-1-\alpha<0$ ), Theorem 3.1 (1) (resp., (2)) yields

$$
\left(\frac{|p-1-\alpha|}{p}\right)^{p} \int_{\Omega} \frac{F^{p}\left(\nabla \rho_{\varepsilon}\right)}{\rho_{\varepsilon}^{p-\alpha}}|u|^{p} d \mathfrak{m} \leq \int_{\Omega} \rho_{\varepsilon}^{\alpha} \max \left\{F^{* p}( \pm d u)\right\} d \mathfrak{m} .
$$

We point out that (4.2) implies (4.1).

Case 1. Suppose $\alpha \geq 0$. Since $\frac{F^{p}\left(\nabla \rho_{\varepsilon}\right)}{\rho_{\varepsilon}^{p-\alpha}}|u|^{p} \in L^{1}(\Omega)$ and

$$
\rho_{\varepsilon}^{\alpha} \max \left\{F^{* p}( \pm d u)\right\} \leq(\rho+1)^{\alpha} \max \left\{F^{* p}( \pm d u)\right\} \in L^{1}(\Omega),
$$

(4.2) together with Fatou's lemma and Lebesgue's dominated convergence theorem yields (4.1). That is,

$$
\begin{aligned}
& \left(\frac{|p-1-\alpha|}{p}\right)^{p} \int_{\Omega}|u|^{p} \frac{F^{p}(\nabla \rho)}{\rho^{p-\alpha}} d \mathfrak{m}=\left(\frac{|p-1-\alpha|}{p}\right)^{p} \int_{\Omega} \liminf _{\varepsilon \rightarrow 0^{+}}\left(|u|^{p} \frac{F^{p}\left(\nabla \rho_{\varepsilon}\right)}{\rho_{\varepsilon}^{p-\alpha}}\right) d \mathfrak{m} \\
\leq & \liminf _{\varepsilon \rightarrow 0^{+}}\left(\frac{|p-1-\alpha|}{p}\right)^{p} \int_{\Omega}\left(|u|^{p} \frac{F^{p}\left(\nabla \rho_{\varepsilon}\right)}{\rho_{\varepsilon}^{p-\alpha}}\right) d \mathfrak{m} \leq \liminf _{\varepsilon \rightarrow 0^{+}} \int_{\Omega} \rho_{\varepsilon}^{\alpha} \max \left\{F^{*}( \pm d u)\right\} d \mathfrak{m} \\
= & \int_{\Omega} \rho^{\alpha} \max \left\{F^{* p}( \pm d u)\right\} d \mathfrak{m} .
\end{aligned}
$$

Case 2. Suppose $\alpha<0$. In this case, $\rho_{\varepsilon}^{\alpha} \max \left\{F^{* p}( \pm d u)\right\} \in L^{1}(\Omega)$ and

$$
\rho_{\varepsilon_{2}}^{\alpha} \max \left\{F^{* p}( \pm d u)\right\} \leq \rho_{\varepsilon_{1}}^{\alpha} \max \left\{F^{* p}( \pm d u)\right\}, \text { if } \varepsilon_{1} \leq \varepsilon_{2} .
$$

Now (4.2) together with Fatou's lemma and Lebesgue's monotone convergence Theorem yields (4.1).

Step 2. From Step 1, it remains to show that $f_{X} \leq \operatorname{div} X$ in the weak sense, that is, for any nonnegative function $u \in C_{0}^{\infty}(\Omega)$, one has

$$
(p-1-\alpha)^{2} \int_{\Omega} \frac{F^{p}\left(\nabla \rho_{\varepsilon}\right)}{\rho_{\varepsilon}^{p-\alpha}} u d \mathfrak{m} \leq(p-1-\alpha) \int_{\Omega} \frac{F^{p-2}\left(\nabla \rho_{\varepsilon}\right)\left\langle\nabla \rho_{\varepsilon}, d u\right\rangle}{\rho_{\varepsilon}^{p-1-\alpha}} d \mathfrak{m} .
$$

In order to prove this, choose an open set $U$ with $\operatorname{supp}(u) \subset U \subset \subset \Omega$. Let $W^{1, p}(U)$ be the completion of $C_{0}^{\infty}(U)$ with respect to the norm $\|u\|_{W^{1, p}(U)}:=\left(\int_{U}|u|^{p} d \mathfrak{m}+\int_{U} F^{* p}(d u) d \mathfrak{m}\right)^{1 / p}$. Since $\bar{U}$ is compact, $W^{1, p}(U)$ is a Sobolev space in the sense of Hebey [17, Definition 2.1].

For any $k>\varepsilon$, define $\rho_{k \varepsilon}:=\inf \left\{\left.\rho_{\varepsilon}\right|_{U}, k\right\} \in L^{1}(U)$. It is easy to check $\ln \rho_{k \varepsilon} \in W^{1, p}(U)$ and hence, there is a sequence $\phi_{n} \in C^{\infty}(U)$ such that

$$
\left\|\phi_{n}-\ln \rho_{k \varepsilon}\right\|_{W^{1, p}(U)} \rightarrow 0, \phi_{n} \rightarrow \ln \rho_{k \varepsilon} \text { pointwise a.e., } \ln \varepsilon \leq \phi_{n} \leq \ln k .
$$


Let $\psi_{n}:=\exp \left(\phi_{n}\right)$. Then $\psi_{n} \in C^{\infty}(U)$ with $\varepsilon \leq \psi_{n} \leq k$,

$$
\int_{U}\left|\ln \psi_{n}-\ln \rho_{k \varepsilon}\right|^{p} d \mathfrak{m} \rightarrow 0, \int_{U} F^{* p}\left(\frac{d \psi_{n}}{\psi_{n}}-\frac{d \rho_{k \varepsilon}}{\rho_{k \varepsilon}}\right) d \mathfrak{m} \rightarrow 0 .
$$

Now we choose $v_{n}:=u / \psi_{n}^{p-1-\alpha} \in C_{0}^{\infty}(\Omega)$ as test functions. Since $-(p-1-\alpha) \Delta_{p} \rho \geq 0$ in the weak sense, one has

$$
(p-1-\alpha)^{2} \int_{\Omega} \frac{F^{p-2}(\nabla \rho)\left\langle\nabla \rho, d \psi_{n}\right\rangle}{\psi_{n}^{p-\alpha}} u d \mathfrak{m} \leq(p-1-\alpha) \int_{\Omega} \frac{F^{p-2}(\nabla \rho)\langle\nabla \rho, d u\rangle}{\psi_{n}^{p-1-\alpha}} d \mathfrak{m} .
$$

In the following, we derive (4.3) from (4.5).

Case 1. Suppose $p-1-\alpha>0$. We study the right hand side of (4.5) first. By (2.2), one has

$$
\left|\frac{F^{p-2}(\nabla \rho)}{\psi_{n}^{p-1-\alpha}}\langle\nabla \rho, d u\rangle\right| \leq \lambda_{F}(U) \frac{F^{p-1}(\nabla \rho)}{\varepsilon^{p-1-\alpha}} F^{*}(d u) \in L^{1}(\Omega) .
$$

Since $\psi_{n} \rightarrow \rho_{k \varepsilon}$ pointwise a.e., (4.6) together with Lebesgue's dominated convergence theorem yields

$$
\lim _{n \rightarrow \infty} \int_{\Omega} \frac{F^{p-2}(\nabla \rho)}{\psi_{n}^{p-1-\alpha}}\langle\nabla \rho, d u\rangle d \mathfrak{m}=\int_{\Omega} \frac{F^{p-2}(\nabla \rho)}{\rho_{k \varepsilon}^{p-1-\alpha}}\langle\nabla \rho, d u\rangle d \mathfrak{m} .
$$

Now we consider the left hand side of (4.5). Firstly, an argument similar to the one above furnishes

$$
\lim _{n \rightarrow \infty}\left\|F^{p-2}(\nabla \rho)\left\langle\nabla \rho, \frac{d \rho_{k \varepsilon}}{\rho_{k \varepsilon}}\right\rangle\left(\frac{1}{\psi_{n}^{p-\alpha-1}}-\frac{1}{\rho_{k \varepsilon}^{p-\alpha-1}}\right) u\right\|_{L^{1}(\Omega)}=0 .
$$

Secondly, (2.2) together with the Hölder inequality and (4.4) implies

$$
\begin{aligned}
& \left\|\frac{F^{p-2}(\nabla \rho)}{\psi_{n}^{p-1-\alpha}}\left\langle\nabla \rho, \frac{d \psi_{n}}{\psi_{n}}-\frac{d \rho_{k \varepsilon}}{\rho_{k \varepsilon}}\right\rangle u\right\|_{L^{1}(\Omega)} \\
\leq & \lambda_{F}(U) \frac{\max _{U}|u|}{\varepsilon^{p-1-\alpha}}\left\|F^{p-1}(\nabla \rho)\right\|_{L^{p /(p-1)}(U)}\left\|F^{*}\left(\frac{d \psi_{n}}{\psi_{n}}-\frac{d \rho_{k \varepsilon}}{\rho_{k \varepsilon}}\right)\right\|_{L^{p}(U)} \rightarrow 0, n \rightarrow+\infty,
\end{aligned}
$$

which together with (4.8) yields

$$
\lim _{n \rightarrow \infty} \int_{\Omega} \frac{F^{p-2}(\nabla \rho)}{\psi_{n}^{p-\alpha}}\left\langle\nabla \rho, d \psi_{n}\right\rangle u d \mathfrak{m}=\int_{\Omega} \frac{F^{p-2}(\nabla \rho)}{\rho_{k \varepsilon}^{p-\alpha}}\left\langle\nabla \rho, d \rho_{k \varepsilon}\right\rangle u d \mathfrak{m} .
$$

Now (4.5) together with (4.7) and (4.9) yields

$$
(p-1-\alpha)^{2} \int_{\Omega} \frac{F^{p-2}(\nabla \rho)\left\langle\nabla \rho, d \rho_{k \varepsilon}\right\rangle}{\rho_{k \varepsilon}^{p-\alpha}} u d \mathfrak{m} \leq(p-1-\alpha) \int_{\Omega} \frac{F^{p-2}(\nabla \rho)\langle\nabla \rho, d u\rangle}{\rho_{k \varepsilon}^{p-1-\alpha}} d \mathfrak{m} .
$$

Due to $p-\alpha>1,(2.2)$ implies

$$
\begin{aligned}
& \left|\frac{F^{p-2}(\nabla \rho)\left\langle\nabla \rho, d \rho_{k \varepsilon}\right\rangle}{\rho_{k \varepsilon}^{p-\alpha}} u\right| \leq \frac{F^{p}(\nabla \rho) u}{\varepsilon^{p-\alpha}} \in L^{1}(\Omega), \\
& \left|\frac{F^{p-2}(\nabla \rho)\langle\nabla \rho, d u\rangle}{\rho_{k \varepsilon}^{p-1-\alpha}}\right| \leq \lambda_{F}(U) \frac{F^{p-1}(\nabla \rho) F^{*}(d u)}{\varepsilon^{p-1-\alpha}} \in L^{1}(\Omega),
\end{aligned}
$$

which together with (4.10) and Lebesgue's dominated convergence theorem yield (4.3).

Case 2. Suppose $p-1-\alpha<0$. Since $1 / \psi^{p-1-\alpha} \leq k^{\alpha-p+1}$, by a suitable modification to the argument in Case 1, one gets (4.10) again. Then (4.3) follows from a similar argument if $p-\alpha \geq 0$. Now assume $p-\alpha<0$, in which case we study the left hand side of (4.10) first. Note that

$$
\frac{F^{p-2}(\nabla \rho)\left\langle\nabla \rho, d \rho_{k \varepsilon}\right\rangle}{\rho_{k \varepsilon}^{p-\alpha}} u=\frac{F^{p-2}(\nabla \rho)\left\langle\nabla \rho, d \rho_{\varepsilon}\right\rangle}{\rho_{k \varepsilon}^{p-\alpha}} \chi_{\left\{\rho_{\varepsilon} \leq k\right\}} u=\frac{F^{p}(\nabla \rho)}{\rho_{k \varepsilon}^{p-\alpha}} \chi_{\left\{\rho_{\varepsilon} \leq k\right\}} u,
$$


which implies that $\frac{F^{p-2}(\nabla \rho)\left\langle\nabla \rho, d \rho_{k \varepsilon}\right\rangle}{\rho_{k \varepsilon}^{p-\alpha}} u$ is an increasing sequence of nonnegative functions converging pointwise to $\frac{F^{p-2}(\nabla \rho)\left\langle\nabla \rho, d \rho_{\varepsilon}\right\rangle}{\rho_{\varepsilon}^{p-\alpha}} u$ as $k \rightarrow+\infty$. Hence, Lebesgue's monotone convergence theorem furnishes

$$
\lim _{k \rightarrow+\infty} \int_{\Omega} \frac{F^{p-2}(\nabla \rho)\left\langle\nabla \rho, d \rho_{k \varepsilon}\right\rangle}{\rho_{k \varepsilon}^{p-\alpha}} u d \mathfrak{m}=\int_{\Omega} \frac{F^{p-2}(\nabla \rho)\left\langle\nabla \rho, d \rho_{\varepsilon}\right\rangle}{\rho_{\varepsilon}^{p-\alpha}} u d \mathfrak{m} .
$$

Now we study the right hand side of (4.10). The Hölder inequality together with Condition (2) (i.e., $\left.F^{p}(\nabla \rho) / \rho^{p-\alpha}, \rho^{\alpha} \in L_{\text {loc }}^{1}(\Omega)\right)$ yields

$$
\left\|\left(\frac{F^{p}(\nabla \rho)}{\rho_{k \varepsilon}^{p-\alpha}}\right)^{\frac{1}{p^{\prime}}}\left(\rho_{k \varepsilon}^{\alpha}\right)^{\frac{1}{p}}\right\|_{L^{1}(U)} \leq\left\|\frac{F^{p}(\nabla \rho)}{\rho_{k \varepsilon}^{p-\alpha}}\right\|_{L^{1}(U)}^{\frac{1}{p^{\prime}}}\left\|\rho_{k \varepsilon}^{\alpha}\right\|_{L^{1}(U)}^{\frac{1}{p}}<+\infty,
$$

where $p^{\prime}=p /(p-1)$. Therefore, we have

$$
\left|\frac{F^{p-2}(\nabla \rho)\langle\nabla \rho, d u\rangle}{\rho_{k \varepsilon}^{p-1-\alpha}}\right| \leq \lambda_{F}(U) F^{*}(d u) \frac{F^{p-1}(\nabla \rho)}{\rho_{k \varepsilon}^{\frac{p-\alpha}{p^{\prime}}}} \frac{1}{\rho_{k \varepsilon}^{-\frac{\alpha}{p}}} \leq C\left(\frac{F^{p}(\nabla \rho)}{\rho_{k \varepsilon}^{p-\alpha}}\right)^{\frac{1}{p^{\prime}}}\left(\rho_{k \varepsilon}^{\alpha}\right)^{\frac{1}{p}} \in L^{1}(U),
$$

which together with Lebesgue's dominated convergence theorem furnishes

$$
\lim _{k \rightarrow+\infty} \int_{\Omega} \frac{F^{p-2}(\nabla \rho)\langle\nabla \rho, d u\rangle}{\rho_{k \varepsilon}^{p-1-\alpha}} d \mathfrak{m}=\int_{\Omega} \frac{F^{p-2}(\nabla \rho)\langle\nabla \rho, d u\rangle}{\rho_{\varepsilon}^{p-1-\alpha}} d \mathfrak{m} .
$$

Now (4.3) follows from (4.10), (4.11) and (4.12).

Remark 4. For $p>\alpha$, the above theorem implies $\frac{F^{p}(\nabla \rho)}{\rho^{p-\alpha}} \in L_{\text {loc }}^{1}(\Omega)$ naturally.

4.2. Best constant and Brezis-Vázquez improvement. Suppose the assumption of Theorem 4.1 holds and additionally assume that $p-1-\alpha \neq 0, \rho>0$ a.e. and $d \rho \neq 0$ a.e.. Then one can define a norm on $C_{0}^{\infty}(\Omega)$ by

$$
|u|_{D}:=\left(\int_{\Omega} \rho^{\alpha} \max \left\{F^{* p}( \pm d u)\right\} d \mathfrak{m}\right)^{\frac{1}{p}} .
$$

Denote by $D^{1, p}\left(\Omega, \rho^{\alpha}\right)$ the closure of $C_{0}^{\infty}(\Omega)$ with respect to the norm $|\cdot|_{D}$.

Remark 5. In fact, $D^{1, p}\left(\Omega, \rho^{\alpha}\right)$ is the completion of $C_{0}^{\infty}(\Omega)$ with respect to the norm

$$
\|u\|_{D}:=\left(\int_{\Omega} \frac{F^{p}(\nabla \rho)}{\rho^{p-\alpha}}|u|^{p} d \mathfrak{m}+\int_{\Omega} \rho^{\alpha} \max \left\{F^{* p}( \pm d u)\right\} d \mathfrak{m}\right)^{\frac{1}{p}} .
$$

In view of Theorem $4.1,|\cdot|_{D}$ is equivalent to $\|\cdot\|_{D}$.

Proposition 4.2. Let $(M, F, d \mathfrak{m})$ be a forward complete $F M M M$ and let $\Omega \subset M$ be a natural domain. Given $p>1$ with $p-1-\alpha \neq 0$, suppose $\rho \in W_{\mathrm{loc}}^{1, p}(\Omega)$ satisfies the following properties:

(i) $-(p-1-\alpha) \Delta_{p} \rho \geq 0$ in the weak sense;

(ii) $\frac{F^{p}(\nabla \rho)}{\rho^{p-\alpha}}, \rho^{\alpha} \in L_{\text {loc }}^{1}(\Omega)$ if $\alpha>p$.

(iii) $\rho>0$ a.e. and $d \rho \neq 0$ a.e. with $F^{*}(d \rho) \geq F^{*}(-d \rho)$; Set

$$
\mathfrak{C}_{p, \alpha}(\Omega):=\inf _{u \in D^{1, p}\left(\Omega, \rho^{\alpha}\right) \backslash\{0\}} \frac{\int_{\Omega} \rho^{\alpha} \max \left\{F^{* p}( \pm d u)\right\} d \mathfrak{m}}{\int_{\Omega} \frac{F^{p}(\nabla \rho)}{\rho^{p-\alpha}}|u|^{p} d \mathfrak{m}} .
$$

Thus, if $\rho^{\frac{p-1-\alpha}{p}} \in D^{1, p}\left(\Omega, \rho^{\alpha}\right)$, then

$$
\mathfrak{C}_{p, \alpha}(\Omega)=\left(\frac{|p-1-\alpha|}{p}\right)^{p}
$$

and $C \rho^{\frac{p-1-\alpha}{p}}$ is an extremal, where $C \in \mathbb{R} \backslash\{0\}$. 
Proof. Theorem 4.1 implies $\mathfrak{C}_{p, \alpha}(\Omega) \geq\left(\frac{|p-1-\alpha|}{p}\right)^{p}$. Now set $\varphi=C \rho^{\frac{p-\alpha-1}{p}}$. A direct calculation together with the assumption yields

$$
\rho^{\alpha} \max \left\{F^{* p}( \pm d \varphi)\right\}=|C|^{p}\left(\frac{|p-\alpha-1|}{p}\right)^{p} \frac{F^{* p}(d \rho)}{\rho} \in L^{1}(\Omega),
$$

which furnishes

$$
\int_{\Omega} \rho^{\alpha} \max \left\{F^{* p}( \pm d \varphi)\right\} d \mathfrak{m}=\left(\frac{|p-\alpha-1|}{p}\right)^{p} \int_{\Omega} \frac{F^{p}(\nabla \rho)}{\rho^{p-\alpha}}|\varphi|^{p} d \mathfrak{m}
$$

Hence, it follows that $\mathfrak{C}_{p, \alpha}(\Omega) \leq\left(\frac{|p-1-\alpha|}{p}\right)^{p}$.

We also have the following Brezis-Vázquez improvement for $p=2$ and $\alpha=0$.

Proposition 4.3. Let $(M, F, d \mathfrak{m})$ be a forward complete $F M M M$ with finite uniformity constant $\Lambda_{F}$ and let $\Omega \subset M$ be a natural domain. Suppose that $\rho \in W_{\mathrm{loc}}^{1,2}(\Omega)$ satisfies $\rho>0$ a.e. and $-\Delta \rho \geq 0$ in the weak sense. Set $\Theta(\Omega)$ as

$$
\Theta(\Omega):=\inf _{u \in C_{0}^{1}(\Omega) \backslash\{0\}} \frac{\int_{\Omega} \rho \min \left\{F^{* 2}( \pm d u)\right\} d \mathfrak{m}}{\int_{\Omega} \rho u^{2} d \mathfrak{m}} .
$$

Then we have

$$
\int_{\Omega} \max \left\{F^{* 2}( \pm d u)\right\} d \mathfrak{m} \geq \frac{1}{4} \int_{\Omega} \frac{u^{2}}{\rho^{2}} F^{2}(\nabla \rho) d \mathfrak{m}+\frac{\Theta(\Omega)}{\Lambda_{F}} \int_{\Omega} u^{2} d \mathfrak{m}, \forall u \in C_{0}^{\infty}(\Omega) .
$$

In particular, if $\rho^{1 / 2} \notin D^{1,2}(\Omega)$ but $\mathfrak{C}_{2,0}(\Omega)=1 / 4$, then the best constant $\mathfrak{C}_{2,0}(\Omega)$ is not achieved.

Proof. Given $u \in C_{0}^{1}(\Omega)$ and $\varepsilon \in(0,1)$, set $\rho_{\varepsilon}:=\rho+\varepsilon$ and $v:=u / \rho_{\varepsilon}^{\frac{1}{2}}$. Let $\Omega_{0}:=\{x \in \Omega: v(x)=0\}$, $\Omega_{+}:=\{x \in \Omega: v(x)>0\}, \Omega_{-}:=\{x \in \Omega: v(x)<0\}$. Set $\xi:=\frac{1}{2} v \rho_{\varepsilon}^{-\frac{1}{2}} d \rho_{\varepsilon}$ and $\eta:=\rho_{\varepsilon}^{\frac{1}{2}} d v$. Thus, $d u=\xi+\eta$. Using (2.3), on $\Omega_{+} \cup \Omega_{0}$ we have

$$
\max \left\{F^{* 2}( \pm d u)\right\} \geq F^{* 2}(d u) \geq \frac{1}{4} v^{2} \rho_{\varepsilon}^{-1} F^{* 2}\left(d \rho_{\varepsilon}\right)+v g_{d \rho_{\varepsilon}}^{*}\left(d \rho_{\varepsilon}, d v\right)+\frac{\rho_{\varepsilon}}{\Lambda_{F}} F^{* 2}(d v),
$$

which yields

$$
\begin{aligned}
& \int_{\Omega_{+} \cup \Omega_{0}} \max \left\{F^{* 2}( \pm d u)\right\} d \mathfrak{m}-\frac{1}{4} \int_{\Omega_{+} \cup \Omega_{0}} \frac{u^{2}}{\rho_{\varepsilon}^{2}} F^{* 2}\left(d \rho_{\varepsilon}\right) d \mathfrak{m} \\
\geq & \frac{1}{2} \int_{\Omega_{+} \cup \Omega_{0}}\left\langle\nabla \rho, d v^{2}\right\rangle d \mathfrak{m}+\frac{1}{\Lambda_{F}} \int_{\Omega_{+} \cup \Omega_{0}} \rho_{\varepsilon} F^{* 2}(d v) d \mathfrak{m} .
\end{aligned}
$$

A similar argument on $\Omega_{-}$furnishes

$$
\begin{aligned}
& \int_{\Omega_{-}} \max \left\{F^{* 2}( \pm d u)\right\} d \mathfrak{m}-\frac{1}{4} \int_{\Omega_{-}} \frac{u^{2}}{\rho_{\varepsilon}^{2}} F^{* 2}\left(d \rho_{\varepsilon}\right) d \mathfrak{m} \\
\geq & \frac{1}{2} \int_{\Omega_{-}}\left\langle\nabla \rho, d v^{2}\right\rangle d \mathfrak{m}+\frac{1}{\Lambda_{F}} \int_{\Omega_{-}} \rho_{\varepsilon} F^{* 2}(-d v) d \mathfrak{m},
\end{aligned}
$$

which together with (4.14) and $-\Delta \rho \geq 0$ (in the weak sense) yields

$$
\begin{aligned}
& \int_{\Omega} \max \left\{F^{* 2}( \pm d u)\right\} d \mathfrak{m}-\frac{1}{4} \int_{\Omega} \frac{u^{2}}{\rho_{\varepsilon}^{2}} F^{* 2}(d \rho) d \mathfrak{m} \\
\geq & \frac{1}{\Lambda_{F}} \int_{\Omega} \rho_{\varepsilon} F^{* 2}(d|v|) d \mathfrak{m} \geq \frac{1}{\Lambda_{F}} \int_{\Omega} \rho \min \left\{F^{* 2}( \pm d v)\right\} d \mathfrak{m} \geq \frac{\Theta(\Omega)}{\Lambda_{F}} \int_{\Omega} \frac{\rho}{\rho_{\varepsilon}} u^{2} d \mathfrak{m} .
\end{aligned}
$$

Note that Remark 4 implies $F^{2}(\nabla \rho) / \rho^{2} \in L_{\text {loc }}^{1}(\Omega)$ and hence,

$$
\left|\frac{u^{2}}{\rho_{\varepsilon}^{2}} F^{* 2}(d \rho)\right| \leq \frac{u^{2}}{\rho^{2}} F^{* 2}(d \rho) \in L^{1}(\Omega),
$$

which together with Lebesgue's dominated convergence theorem and (4.15) yields (4.13). 
Now suppose $\rho^{1 / 2} \notin D^{1,2}(\Omega)$ but $\mathfrak{C}_{2,0}(\Omega)=1 / 4$. Thus, from (4.15) and Lebesgue's dominated convergence theorem, we have

$$
\int_{\Omega} \max \left\{F^{* 2}( \pm d u)\right\} d \mathfrak{m}-\frac{1}{4} \int_{\Omega} \frac{u^{2}}{\rho^{2}} F^{* 2}(d \rho) d \mathfrak{m} \geq \frac{1}{\Lambda_{F}} \int_{\Omega} \rho F^{* 2}(d|v|) d \mathfrak{m}>0
$$

which implies the nonexistence of minimizers in $D^{1,2}(\Omega)$.

\section{Appendix A. Two lemmas}

Lemma A.1. Let $(M, o, F, d \mathfrak{m}), \Omega, p, \beta$ be as in Definition 3.5. If $u$ is a globally Lipschitz function on $M$ with compact support in $\Omega$, then $u \in D^{1, p}\left(\Omega, r^{p+\beta}\right)$.

Proof. Since $\operatorname{supp}(u)$ is compact, there exist a coordinate covering $\left\{\left(U_{k}, \phi_{k}\right)\right\}_{k=1}^{N<\infty}$ of $\operatorname{supp}(u)$ and a constant $C \geq 1$ such that for each $k, U_{k} \subset \subset \Omega, \phi_{k}\left(U_{k}\right)=\mathbb{B}_{\mathbf{0}}(1)$ and

$$
C^{-1} d \mathrm{vol} \leq\left. d \mathfrak{m}\right|_{U_{k}} \leq C d \text { vol, } \quad C^{-1} \leq \frac{F^{*}(\omega)}{\left\|\phi_{k *}^{-1} \omega\right\|} \leq C, \text { for any } \omega \in T^{*} U_{k} \backslash\{0\},
$$

where $d$ vol and $\|\cdot\|$ are the Lebesgue measure and the Euclidean norm on the unit ball $\mathbb{B}_{\mathbf{0}}(1)$, respectively.

Choose a number $q>1$ such that $\beta q /(q-1)>-n$ if $\beta>-n$. By Lemma 3.2 and the construction above, one can easily verify $\int_{U_{k}} r^{\beta q /(q-1)} d \mathfrak{m}<\infty$ for each $k$.

On the other hand, let $\left\{\eta_{k}\right\}$ be a smooth partition of unity subordinate to $\left\{U_{k}\right\}$. Thus, $\left(\eta_{k} u\right) \circ \phi_{k}^{-1}$ is a globally Lipschitz function on $\mathbb{B}_{\mathbf{0}}(1)$ with respect to the Euclidean distance and hence, $\left(\eta_{k} u\right) \circ \phi_{k}^{-1}$ belongs to the Sobolev space $W^{1, p q}\left(\mathbb{B}_{\mathbf{0}}(1)\right)$. Meyers-Serrin's theorem then yields a sequence $v_{k_{j}} \in C_{0}^{\infty}\left(\mathbb{B}_{\mathbf{0}}(1)\right)$ with $\lim _{j \rightarrow+\infty}\left\|v_{k_{j}}-\left(\eta_{k} u\right) \circ \phi_{k}^{-1}\right\|_{W^{1, p q}\left(\mathbb{B}_{\mathbf{0}}(1)\right)}=0$. Therefore, we have $v_{k_{j}} \circ \phi_{k} \in C_{0}^{\infty}(\Omega)$ with $\operatorname{supp}\left(v_{k_{j}} \circ \phi_{k}\right) \subset U_{k}$, which together with the Hölder inequality and (A.1) implies

$$
\begin{aligned}
\int_{\Omega}\left|v_{k_{j}} \circ \phi_{k}-\left(\eta_{k} u\right)\right|^{p} r^{\beta} d \mathfrak{m} & \leq\left(\left.C \int_{\mathbb{B}_{\mathbf{0}}(1)}\left|v_{k_{j}}-\left(\eta_{k} u\right) \circ \phi_{k}^{-1}\right|\right|^{p q} d \mathrm{vol}\right)^{\frac{1}{q}}\left(\int_{U_{k}} r^{\frac{\beta q}{q-1}} d \mathfrak{m}\right)^{\frac{q-1}{q}} \\
& \leq C^{\frac{1}{q}}\left\|v_{k_{j}}-\left(\eta_{k} u\right) \circ \phi_{k}^{-1}\right\|_{W^{1, p q}\left(\mathbb{B}_{\mathbf{0}}(1)\right)}^{p}\left(\int_{U_{k}} r^{\frac{\beta q}{q-1}} d \mathfrak{m}\right)^{\frac{q-1}{q}} \rightarrow 0, \text { as } j \rightarrow+\infty .
\end{aligned}
$$

Similarly, one can prove $\lim _{j \rightarrow+\infty} \int_{\Omega} F^{* p}\left(d\left(v_{k_{j}} \circ \phi_{k}\right)-d\left(\eta_{k} u\right)\right) r^{\beta+p} d \mathfrak{m}=0$. Therefore, $\left\|v_{k_{j}} \circ \phi_{k}-\left(\eta_{k} u\right)\right\|_{D} \rightarrow$ 0 and $\left(\eta_{k} u\right) \in D^{1, p}\left(\Omega, r^{p+\beta}\right)$. We conclude the proof by $u=\sum_{k=1}^{N}\left(\eta_{k} u\right)$.

Lemma A.2. Let $(M, o, F, d \mathfrak{m})$ be an $n$-dimensional forward complete PFMMM with $\mathbf{R i c}_{\infty} \geq(n-1) K$ and $\mathbf{S}_{o}^{+} \geq-a$, where $a \geq 0$. Set $\frac{\pi}{\sqrt{K}}:=+\infty$ if $K \leq 0$. Let $(t, y)$ denote the polar coordinate system around o. Then we have

$$
\Delta t \leq(n-1) \frac{\mathfrak{s}_{K}^{\prime}(t)}{\mathfrak{s}_{K}(t)}+a, \text { for any } y \in S_{o} M, 0<t<\min \left\{i_{y}, \frac{\pi}{2 \sqrt{K}}\right\}
$$

which implies

$$
\hat{\sigma}_{o}(t, y) \leq e^{-\tau(y)+a t} \mathfrak{s}_{K}^{n-1}(t), \quad \text { for any } y \in S_{o} M, 0<t<\min \left\{i_{y}, \frac{\pi}{2 \sqrt{K}}\right\} .
$$

where $\hat{\sigma}_{o}(t, y)$ is defined as in (2.7) and $\mathfrak{s}_{K}(t)$ is the solution of $f^{\prime \prime}+K f=0$ with $f(0)=0$ and $f^{\prime}(0)=1$. Hence,

$$
\mathfrak{m}\left(B_{o}^{+}(r)\right) \leq \int_{S_{o} M} e^{-\tau(y)} d \nu_{o}(y) \int_{0}^{r} e^{a t} \mathfrak{s}_{K}^{n-1}(t) d t, \text { for } 0<r<\min \left\{i_{y}, \frac{\pi}{2 \sqrt{K}}\right\} .
$$

Proof. The proof is similar to that of Wei and Wylie [38, Theorem 1.1]. First, fix $y \in S_{o} M$ and set

$$
H(t):=\frac{\partial}{\partial t} \log \sqrt{\operatorname{det} g \nabla t}, \tau(t):=\tau(\nabla t), \mathbf{S}(t):=\mathbf{S}(\nabla t)=\frac{d}{d t} \tau(t) .
$$


A standard argument (cf. Wu $[39,(4.5)])$ yields $\Delta t=H(t)-\mathbf{S}(t)$ and

$$
\frac{d}{d t} H \leq-\mathbf{R i c}(\nabla t)-\frac{H^{2}}{n-1} .
$$

Also set $H_{K}(t):=(n-1) \frac{\mathfrak{s}_{K}^{\prime}(t)}{\mathfrak{s}_{K}(t)}$. By (A.2), one has

$$
\begin{aligned}
& \frac{d}{d t}\left(H(t) \mathfrak{s}_{K}^{2}(t)\right) \leq 2 \mathfrak{s}_{K}^{\prime}(t) \mathfrak{s}_{K}(t) H(t)-\mathfrak{s}_{K}^{2}(t)\left(\mathbf{R i c}(\nabla t)+\frac{H^{2}}{n-1}\right) \\
= & -\left(\frac{\mathfrak{s}_{K}(t) H(t)}{\sqrt{n-1}}-\sqrt{n-1} \mathfrak{s}_{K}^{\prime}(t)\right)^{2}+(n-1)\left(\mathfrak{s}_{K}^{\prime}(t)\right)^{2}-\mathfrak{s}_{K}^{2}(t) \mathbf{R i c}(\nabla t) \\
\leq & (n-1)\left(\mathfrak{s}_{K}^{\prime}(t)\right)^{2}-\mathfrak{s}_{K}^{2}(t)\left(\mathbf{R i c}_{\infty}(\nabla t)-\frac{d}{d t} \mathbf{S}(t)\right) \\
\leq & (n-1)\left(\mathfrak{s}_{K}^{\prime}(t)\right)^{2}-(n-1) K \mathfrak{s}_{K}^{2}(t)+\mathfrak{s}_{K}^{2}(t) \frac{d}{d t} \mathbf{S}(t) \\
= & \frac{d}{d t}\left(H_{K}(t) \mathfrak{s}_{K}^{2}(t)\right)+\mathfrak{s}_{K}^{2}(t) \frac{d}{d t} \mathbf{S}(t),
\end{aligned}
$$

Since $\mathbf{S}_{O} \geq-a$, integrating by parts on the above inequality, we get

$$
\begin{aligned}
& \mathfrak{s}_{K}^{2}(t) \Delta t=\mathfrak{s}_{K}^{2}(t)(H(t)-\mathbf{S}(t)) \leq \mathfrak{s}_{K}^{2}(t) H_{K}(t)-\int_{0}^{t} \frac{d}{d s}\left(\mathfrak{s}_{K}^{2}(s)\right) \mathbf{S}(s) d s \\
\leq & \mathfrak{s}_{K}^{2}(t) H_{K}(t)+a \int_{0}^{t} \frac{d}{d s}\left(\mathfrak{s}_{K}^{2}(s)\right) d s=\mathfrak{s}_{K}^{2}(t) H_{K}(t)+a \mathfrak{s}_{K}^{2}(t) .
\end{aligned}
$$

Hence, $\Delta t \leq H_{K}(t)+a$, which implies

$$
\frac{\partial}{\partial t} \log \hat{\sigma}_{o}(t, y)=\Delta t \leq(n-1) \frac{\mathfrak{s}_{K}^{\prime}(t)}{\mathfrak{s}_{K}(t)}+a=\frac{\partial}{\partial t} \log \left[e^{a t} \mathfrak{s}_{K}^{n-1}(t)\right] .
$$

Then the estimates of $\hat{\sigma}_{o}(t, y)$ and $\mathfrak{m}\left(B_{o}^{+}(r)\right)$ follow from a standard argument (cf. Zhao et al. [44]).

Remark 6. By a different method, Yin [42] obtained the theorem above in the case when the PFMMM is equipped by the Busemann-Hausdroff measure and satisfies $\mathbf{R i c} \mathbf{c}_{\infty} \geq(n-1) K$ and $\mathbf{S}_{o}^{+} \geq-a(a>0)$.

\section{Appendix B. Weighted Sobolev space}

Let $(M, o, F, d \mathfrak{m})$ be an $n$-dimensional closed reversible RFMMM and set $r(x):=d_{F}(o, x)$. Given $p \in(1,+\infty)$ and $\beta<-n$ with $p+\beta>-n$, by Lemma 3.2, we define a norm on $C_{0}^{\infty}(M)=C^{\infty}(M)$ as

$$
\|u\|_{p, \beta}:=\left(\int_{M}|u|^{p} r^{p+\beta} d \mathfrak{m}+\int_{M} F^{* p}(d u) r^{p+\beta} d \mathfrak{m}\right)^{\frac{1}{p}} .
$$

The weighted Sobolev space $W^{1, p}\left(M, r^{p+\beta}\right)$ is defined as

$$
W^{1, p}\left(M, r^{p+\beta}\right):=\bar{C}_{0}^{\infty}(M) \cdot \|_{p, \beta} .
$$

In particular, $W^{1, p}\left(M, r^{0}\right)=: W^{1, p}(M)$, i.e., the standard Sobolev space in the sense of Hebey [17, Definition 2.1].

We also define the weighted $L^{p}$-space $L^{p}\left(M, r^{p+\beta}\right)$ (resp., $\left.L^{p}\left(T M, r^{p+\beta}\right)\right)$ as the completion of $C^{\infty}(M)$ (resp., $\Gamma^{\infty}\left(T^{*} M\right)$, i.e., the space of the smooth sections of the cotangent bundle) under the norm

$$
[u]_{p, \beta}:=\left(\int_{M}|u|^{p} r^{p+\beta} d \mathfrak{m}\right)^{\frac{1}{p}}\left(\operatorname{resp} .,[\omega]_{p, \beta}:=\left(\int_{M} F^{* p}(\omega) r^{p+\beta} d \mathfrak{m}\right)^{\frac{1}{p}}\right) .
$$

And set $L^{p}(M):=L^{p}\left(M, r^{0}\right)$ and $L^{p}(T M):=L^{p}\left(T M, r^{0}\right)$. 
Lemma B.1. If $u \in W^{1, p}\left(M, r^{p+\beta}\right)$, then $u \in W^{1,1}(M)$. Moreover, the differential $\varpi$ of $u$ in $W^{1, p}\left(M, r^{p+\beta}\right)$ is the distributional derivative of $u$, i.e., $\varpi \in L^{1}(T M)$ and

$$
\int_{M}\langle X, \varpi\rangle d \mathfrak{m}=-\int_{M} u \operatorname{div} X d \mathfrak{m}, \text { for any smooth vector field } X .
$$

Proof. Since $(\beta+p) /(1-p)>-n$, Lemma 3.2 implies that $r^{\frac{\beta+p}{1-p}}$ is integrable. Given $f \in L^{p}\left(M, r^{\beta+p}\right)$, the Hölder inequality yields

$$
\int_{M}|f| d \mathfrak{m}=\int_{M}|f| r^{\frac{\beta+p}{p}} r^{-\frac{\beta+p}{p}} d \mathfrak{m} \leq\left(\int_{M}|f|^{p} r^{p+\beta} d \mathfrak{m}\right)^{\frac{1}{p}}\left(\int_{M} r^{\frac{p+\beta}{1-p}} d \mathfrak{m}\right)^{\frac{p-1}{p}} .
$$

Consequently, if $u \in W^{1, p}\left(M, r^{p+\beta}\right)$, (B.1) implies $u \in L^{1}(M)$ and its differential $\varpi \in L^{1}(T M)$. On the other hand, there exist a sequence $u_{j} \in C_{0}^{\infty}(M)$ such that $\left[u_{j}-u\right]_{p, \beta} \rightarrow 0$ and $\left[d u_{j}-\varpi\right]_{p, \beta} \rightarrow 0$. Thus, for any smooth vector field $X,(B .1)$ together with the compactness of $M$ yields

$$
\begin{aligned}
& \left|\int_{M}\langle X, \varpi\rangle-(-u \operatorname{div} X) d \mathfrak{m}\right|=\left|\int_{M}\langle X, \varpi\rangle-\left\langle X, d u_{j}\right\rangle+\left\langle X, d u_{j}\right\rangle-(-u \operatorname{div} X) d \mathfrak{m}\right| \\
\leq & \int_{M}\left|\left\langle X, \varpi-d u_{j}\right\rangle\right| d \mathfrak{m}+\int_{M}\left|\left(u_{j}-u\right) \operatorname{div} X\right| d \mathfrak{m} \\
\leq & \max _{M} F(X) \int_{M} F^{*}\left(\varpi-d u_{j}\right) d \mathfrak{m}+\max _{M}|\operatorname{div} X| \int_{M}\left|u_{j}-u\right| d \mathfrak{m} \\
\leq & \left(\max _{M} F(X)+\max _{M}|\operatorname{div} X|\right)\left(\int_{M} r^{\frac{p+\beta}{1-p}} d \mathfrak{m}\right)^{\frac{p-1}{p}}\left(\left[\varpi-d u_{j}\right]_{p, \beta}^{p}+\left[u_{j}-u\right]_{p, \beta}^{p}\right) \rightarrow 0 .
\end{aligned}
$$

Furthermore, (B.1) also implies that $u_{j} \rightarrow u$ in $W^{1,1}(M)$ and hence, the lemma follows.

Lemma B.2. If $u \in W^{1, p}\left(M, r^{\beta+p}\right)$, then $u_{+}:=\max \{u, 0\}, u_{-}:=-\min \{u, 0\}$ and $|u|=u_{+}-u_{-}$are all in $W^{1, p}\left(M, r^{\beta+p}\right)$.

Proof. Since $u_{-}=(-u)_{+}$, it suffices to prove $u_{+} \in W^{1, p}\left(M, r^{\beta+p}\right)$. Choose a sufficiently large constant $q>1$ such that $\frac{q(\beta+p)}{q-1}>-n$. For any $f \in L^{p q}(M)$, the Hölder inequality together with Lemma 3.2 yields

$$
\int_{M}|f|^{p} r^{\beta+p} d \mathfrak{m} \leq\left(\int_{M}|f|^{p q} d \mathfrak{m}\right)^{\frac{1}{q}}\left(\int_{M} r^{\frac{q(\beta+p)}{q-1}} d \mathfrak{m}\right)^{\frac{q-1}{q}} .
$$

First we consider the case when $u \in C_{0}^{\infty}(M)$. The standard theory yields a subsequence $u_{j} \in C_{0}^{\infty}(M)$ such that $u_{j} \rightarrow u_{+}$in $W^{1, p q}(M)$ (cf. Hebey [17, Lemma 2.5]), which together with (B.2) implies $u_{j} \rightarrow u_{+}$ in $W^{1, p}\left(M, r^{p+\beta}\right)$. Hence, $u_{+} \in W^{1, p}\left(M, r^{p+\beta}\right)$.

For the general case (i.e., $u \in W^{1, p}\left(M, r^{\beta+p}\right)$ ), choose a sequence $u_{j} \in C_{0}^{\infty}(M)$ such that $\left\|u_{j}-u\right\|_{p, \beta} \rightarrow 0$. From above, we have $u_{j+}=\max \left\{u_{j}, 0\right\} \in W^{1, p}\left(M, r^{p+\beta}\right)$. Since $\max \{s, t\}=\frac{1}{2}(s+t-|s-t|)$, the triangle inequality yields $\left\|u_{j+}-u_{+}\right\|_{p, \beta} \leq\left\|u_{j}-u\right\|_{p, \beta} \rightarrow 0$. Hence, $u_{+} \in W^{1, p}\left(M, r^{\beta+p}\right)$.

Since $M$ is closed, the following result follows from Lemma B.2 directly.

Corollary B.3. Given $u \in W^{1, p}\left(M, r^{\beta+p}\right)$, then $u_{\epsilon}:=\max \{u-\epsilon, 0\} \in W^{1, p}\left(M, r^{\beta+p}\right)$, for any $\epsilon>0$.

Now set $M_{o}:=M \backslash\{o\}$. Define the weighted Sobolev space $W^{1, p}\left(M_{o}, r^{\beta+p}\right)$ as the completion of $C_{0}^{\infty}\left(M_{o}\right)$ with respect to the norm

$$
\|u\|_{M_{o}, p, \beta}:=\left(\int_{M_{o}}|u|^{p} r^{p+\beta} d \mathfrak{m}+\int_{M_{o}} F^{* p}(d u)^{p} r^{p+\beta} d \mathfrak{m}\right)^{\frac{1}{p}} .
$$

Lemma B.4. If $u \in W^{1, p}\left(M, r^{\beta+p}\right)$ with compact support in $M_{o}$, then $\left.u\right|_{M_{o}} \in W^{1, p}\left(M_{o}, r^{\beta+p}\right)$.

Proof. Since $\operatorname{supp}(u) \subset M_{o}$ is compact, one can choose a cut-off function $\eta \in C_{0}^{\infty}(M)$ such that $\operatorname{supp}(u) \varsubsetneqq$ $\operatorname{supp}(\eta) \subset M_{o}$ and $\left.\eta\right|_{\operatorname{supp}(u)}=1$. 
On the other hand, since $u \in W^{1, p}\left(M, r^{\beta+p}\right)$, there exist a sequence $u_{i} \in C_{0}^{\infty}(M)$ with $\left\|u_{i}-u\right\|_{p, \beta} \rightarrow 0$. Note that if $\left\|\eta u_{i}-\eta u\right\|_{M_{o}, p, \beta} \rightarrow 0$, then $\left.u\right|_{M_{o}}=\eta u \in W^{1, p}\left(M_{o}, r^{\beta+p}\right)$ and the lemma follows. Hence, it suffices to show $\left\|\eta u_{i}-\eta u\right\|_{M_{o}, p, \beta} \rightarrow 0$.

A direct calculation together with the triangle inequality (i.e., $\left.F^{*}\left(\omega_{1}+\omega_{2}\right) \leq F^{*}\left(\omega_{1}\right)+F^{*}\left(\omega_{2}\right)\right)$ furnishes

$$
\begin{aligned}
& \left\|\eta u_{i}-\eta u\right\|_{M_{o}, p, \beta}^{p}=\int_{\operatorname{supp} \eta}\left|\eta u_{i}-\eta u\right|^{p} r^{\beta+p} d \mathfrak{m}+\int_{\operatorname{supp} \eta} F^{* p}\left(d\left(\eta u_{i}-\eta u\right)\right) r^{\beta+p} d \mathfrak{m} \\
\leq & \int_{M}\left|u_{i}-u\right|^{p} r^{\beta+p} d \mathfrak{m}+2^{p}\left[\int_{\operatorname{supp} \eta} F^{* p}\left(\left(u_{i}-u\right) d \eta\right) r^{\beta+p} d \mathfrak{m}+\int_{\operatorname{supp} \eta} F^{* p}\left(\eta d\left(u_{i}-u\right)\right) r^{\beta+p} d \mathfrak{m}\right] \\
\leq & \int_{M}\left|u_{i}-u\right|^{p} r^{\beta+p} d \mathfrak{m}+2^{p}\left[\left\|F^{* p}(d \eta)\right\|_{\infty} \int_{M}\left|u_{i}-u\right|^{p} r^{\beta+p} d \mathfrak{m}+\int_{M} F^{* p}\left(d u_{i}-d u\right) r^{\beta+p} d \mathfrak{m}\right] \\
\leq & {\left[2^{p}\left(\left\|F^{* p}(d \eta)\right\|_{\infty}+1\right)+1\right]\left\|u_{i}-u\right\|_{p, \beta}^{p} \rightarrow 0 . }
\end{aligned}
$$

Proof of Proposition 3.15. Without loss of generality, we may prove the proposition in the case when $u \geq 0$. Thus, Lemma B.2 implies $u=u_{+} \in W^{1, p}\left(M, r^{\beta+p}\right) \cap C(M)$.

For each $\epsilon \in(0,1)$, set $u_{\epsilon}(x):=\max \{u-\epsilon, 0\}$. Since $u$ is continuous with $u(o)=0$, there exists a small $\delta>0$ such that $u_{\epsilon}=0$ in $B_{o}(\delta)$, which implies that $\operatorname{supp}\left(u_{\epsilon}\right)$ is a compact subset of $M_{o}$. Corollary B.3 then yields $\left.u_{\epsilon}\right|_{M_{o}} \in W^{1, p}\left(M_{o}, r^{\beta+p}\right)$. By a direct calculation, we have

$$
\begin{aligned}
&\left\|\left.u\right|_{M_{o}}-\left.u_{\epsilon}\right|_{M_{o}}\right\|_{M_{o}, p, \beta}^{p}=\left\|u-u_{\epsilon}\right\|_{p, \beta}^{p}=\int_{M}\left|u-u_{\epsilon}\right|^{p} r^{p+\beta} d \mathfrak{m}+\int_{M} F^{* p}\left(d\left(u-u_{\epsilon}\right)\right) r^{p+\beta} d \mathfrak{m} \\
& \leq \epsilon^{p} \int_{M} r^{\beta+p} d \mathfrak{m}+\int_{M} \chi_{\{0 \leq u \leq \epsilon\}}|u|^{p} r^{\beta+p} d \mathfrak{m}+\int_{M} \chi_{\{0 \leq u \leq \epsilon\}} F^{* p}(d u) r^{p+\beta} d \mathfrak{m} .
\end{aligned}
$$

Now the assumption together with the dominated convergence theorem yields

$$
\begin{aligned}
& \lim _{\epsilon \rightarrow 0^{+}} \int_{M} \chi_{\{0 \leq u \leq \epsilon\}}|u|^{p} r^{\beta+p} d \mathfrak{m}=\int_{M} \lim _{\epsilon \rightarrow 0^{+}} \chi_{\{0 \leq u \leq \epsilon\}}|u|^{p} r^{\beta+p} d \mathfrak{m}=0, \\
& \lim _{\epsilon \rightarrow 0^{+}} \int_{M} \chi_{\{0 \leq u \leq \epsilon\}} F^{* p}(d u) r^{p+\beta} d \mathfrak{m}=\int_{M} \lim _{\epsilon \rightarrow 0^{+}} \chi_{\{0 \leq u \leq \epsilon\}} F^{* p}(d u) r^{p+\beta} d \mathfrak{m}=0,
\end{aligned}
$$

which imply $\left\|\left.u\right|_{M_{o}}-\left.u_{\epsilon}\right|_{M_{o}}\right\|_{M_{o}, p, \beta}^{p} \rightarrow 0$ as $\epsilon \rightarrow 0^{+}$and hence, $\left.u\right|_{M_{o}} \in W^{1, p}\left(M_{o}, r^{\beta+p}\right)$.

Acknowledgements This work was supported by NNSFC (No. 11761058) and NSFS (No. 19ZR1411700). The author is greatly indebted to Pro. A. Kristály for many useful discussions and helpful comments.

\section{REFERENCES}

[1] Adimurthi, N. Chaudhuri, N. Ramaswamy, An improved Hardy Sobolev inequality and its applications. Proc. Amer. Math. Soc. 130 (2002), 489-505.

[2] J. Alvarez-Paiva, G. Berck, What is wrong with the Hausdorff measure in Finsler spaces. Adv. Math. 204 (2006), 647-663.

[3] J. Alvarez-Paiva, A. C. Thompson, Volumes in normed and Finsler spaces, A Sampler of Riemann-Finsler geometry (Cambridge) (D. Bao, R. Bryant, S.S. Chern, and Z. Shen, eds.), Cambridge University Press, 2004 , pp. 1-49.

[4] P. Baras and L. Cohen, Complete blow-up after Tmax for the solution of a semilinear heat equation. J. Funct. Anal. 71 (1987), 142-174.

[5] D. Bao, S. S. Chern and Z. Shen, An Introduction to Riemannian-Finsler Geometry. GTM 200, Springer-Verlag, 2000.

[6] E. Berchio, D. Ganguly, G. Grillo, Sharp Poincaré-Hardy and Poincaré-Rellich inequalities on the hyperbolic space. J. Funct. Anal. 272 (2017), no. 4, 1661-1703.

[7] H. Brezis, J.-L. Vázquez, Blow-up solutions of some nonlinear elliptic problems. Revista Mat. Univ. Complutense Madrid 10 (1997), 443-469.

[8] G. Carron, Inégalités de Hardy sur les variétés riemanniennes non-compactes. J. Math. Pures Appl. (9) 76 (1997), no. $10,883-891$.

[9] S. S. Chern, Z. Shen, Riemann-Finsler geometry, World Scientific, 2005.

[10] X. Cabré, Y. Martel, Existence versus explosion instantanée pour des équations de la chaleur linéaires avec potentiel singulier. C.R. Acad. Sci. Paris Ser. I Math. 329 (1999) 973-978.

[11] I. Chavel, Riemannian Geometry: A modern introduction, Cambridge Univ., 1993. 
[12] L. D'Ambrosio, Hardy-type inequalities related to degenerate elliptic differential operators. Ann. Sc. Norm. Super. Pisa Cl. Sci. (5), IV (2005), 451-586

[13] L. D'Ambrosio, S. Dipierro, Hardy inequalities on Riemannian manifolds and applications. Ann. Inst. H. Poincaré Anal. Non Linéaire 31 (2014), no. 3, 449-475.

[14] E.-B.Davies, A review of Hardy inequalities. Oper. Theory Adv. Appl. 110 (1998), 55-67.

[15] D. Egloff, Uniform Finsler Hadamard manifolds, Ann. Inst. Henri Poincaré, 66(1997), 323-357.

[16] G. Hardy, G. Pólya, J.E. Littlewood, Inequalities. 2nd edition, Cambridge University, 1952.

[17] E. Hebey, Sobolev Spaces on Riemannian Manifolds. Springer, 1996.

[18] L. Huang, A. Kristály, W. Zhao, Sharp uncertainty principles on general Finsler manifolds, arXiv:1811.08697, 2018 .

[19] A. Kristály, D. Repovš, Quantitative Rellich inequalities on Finsler-Hardamard manifolds. Commun. Contemp. Math. 18 (6) (2016), p.17.

[20] A. Kristály, I. Rudas, Elliptic problems on the ball endowed with Funk-type metrics. Nonlinear Anal. 119 (2015), 199-208.

[21] A. Kristály, A. Szakál, Interpolation between Brezis-Vázquez and Poincaré inequalities on nonnegatively curved spaces: sharpness and rigidities. J. Differential Equations 266 (2019), no. 10, 6621-6646.

[22] B. Li and Z. Shen, On projectively flat fourth root metrics, Canad. Math.Bull, 55(2012), no. 1, $138-145$.

[23] I. Kombe, M. Özaydin, Improved Hardy and Rellich inequalities on Riemannian manifolds. Trans. Amer. Math. Soc. 361 (2009), no. 12, 6191-6203.

[24] I. Kombe, M. Özaydin, Hardy-Poincaré, Rellich and uncertainty principle inequalities on Riemannian manifolds. Trans. Amer. Math. Soc. 365 (2013), no. 10, 5035-5050.

[25] A. Mercaldo, M. Sano, F. Takahshi, Finsler Hardy inequalities, arXiv: 1806.04901v2.

[26] I. Newton, Arithmetica Universalis: Sive de Compositione et Resolutione Arithmetica Liber, 1707.

[27] S. Ohta, K.-T. Sturm, Heat flow on Finsler manifolds, Comm. Pure Appl. Math. 62(10) (2009), $1386-1433$.

[28] S. Ohta, Finsler interpolation inequalities, Calc. Var. Partial Differential Equations, 36(2009), $211-249$.

[29] S. Ohta, Optimal transport and Ricci curvature in Finsler geometry. Probabilistic approach to geometry, 323-342, Adv. Stud. Pure Math., 57, Math. Soc. Japan, Tokyo, 2010.

[30] S. Ohta,Nonlinear geometric analysis on Finsler manifolds, Eur. J. Math. 3(4)(2017), 916-952.

[31] I. Pera, J. L. Vázquez, On the stability or instability of the singular solution of the semilinear heat equation with exponential reaction term. Arch. Rational Mech. Anal. 129 (1995), 201-224.

[32] H. Rademacher, Nonreversible Finsler metrics of positive flag curvature. A sampler of Riemann-Finsler geometry, Cambridge Univ. Press, Cambridge, 2004, pp. 261-302.

[33] Z. Shen, Volume comparison and its applications in Riemann-Finsler geometry. Adv. Math. 128 (1997), no. 2, $306-328$.

[34] Z. Shen, Differential geometry of spray and Finsler spaces. Kluwer Academic Publishers, 2001.

[35] Z. Shen, Lectures on Finsler geometry. World Sci., Singapore, 2001.

[36] J. L. Vazquez, Domain of existence and blowup for the exponential reaction-diffusion equation. Indiana Univ. Math. J. 48 (1999), 677-709.

[37] J. L. Vazque, E. Zuazua, The Hardy inequality and the asymptotic behavior of the heat equation with an inverse-square potential. J. Funct. Anal. 173 (2000), 103-153.

[38] G. Wei, W. Wylie, Comparison geometry for the Bakry-Emery Ricci tensor, J. Differential Geom., 83 (2009), 377-406

[39] B. Y. Wu, On integral Ricci curvature and topology of Finsler manifolds. Int. J. Math., 23(11), (2012), https://doi.org/10.1142/S0129167X1250111X

[40] B. Y. Wu and Y. L. Xin, Comparison theorems in Finsler geometry and their applications. Math. Ann. 337(2007), no. 1, 177-196.

[41] Q. Yang, D. Su, Y. Kong, Hardy inequalities on Riemannian manifolds with negative curvature. Commun. Contemp. Math. 16 (2014), https://doi.org/10.1142/S0219199713500430

[42] S. Yin, Comparison theorems on Finsler manifolds with weighted Ricci curvature bounded below, Front. Math. China, 13(2) (2018), 435-448.

[43] L. Yuan, W. Zhao, Y. Shen, Improved Hardy and Rellich inequalities on nonreversible Finsler manifolds, J. Math. Anal. Appl., 458(2018), 1512-1545.

[44] W. Zhao, Y. Shen, A universal volume comparison theorem for Finsler manifolds and related results. Can. J. Math., 65 (2013), 1401-1435.

Department of Mathematics, East China University of Science and Technology, 200237 Shanghai, China E-mail address: szhao_wei@yahoo.com 